\title{
Capillary wave fluctuations and intrinsic widths of coupled fluid-fluid interfaces: An x-ray scattering study of a wetting film on bulk liquid
}

\author{
Masafumi Fukuto, ${ }^{1,2, *}$ Oleg Gang, ${ }^{1,3}$ Kyle J. Alvine, ${ }^{1}$ and Peter S. Pershan ${ }^{1,4, \dagger}$ \\ ${ }^{1}$ Division of Engineering and Applied Sciences, Harvard University, Cambridge, Massachusetts 02138, USA \\ ${ }^{2}$ Condensed Matter Physics and Materials Science Department, Brookhaven National Laboratory, Upton, New York 11973, USA \\ ${ }^{3}$ Center for Functional Nanomaterials, Brookhaven National Laboratory, Upton, New York 11973, USA \\ ${ }^{4}$ Physics Department, Harvard University, Cambridge, Massachusetts 02138, USA
}

(Received 17 April 2006; revised manuscript received 25 July 2006; published 25 September 2006)

\begin{abstract}
An x-ray specular reflectivity (XR) and off-specular diffuse scattering (XDS) study of the coupled thermal capillary fluctuations and the intrinsic profiles of two interacting fluid-fluid interfaces is presented. The measurements are carried out on complete wetting films of perfluoromethylcyclohexane (PFMC) on the surface of bulk liquid eicosane (C20), as a function of film thickness $30<D<160 \AA$. In order to facilitate the analysis and interpretation of the data with minimal complexity, approximate methods for calculating scattering intensities are developed to take into account the subtleties of thermal diffuse scattering from layered liquid surfaces. With these methods, the calculations of XR/XDS intensities are reduced to a single numerical integration of simple functions in real space. In addition, an analytic expression is derived for small-angle XR that contains Debye-Waller-like factors with effective capillary roughness and takes into account the partial correlations of the two interfaces. The expression for the XR is quantitatively accurate so long as the reflection angle is small enough that the scattering from interfaces is distinguishable from bulk scattering. The results of the XR and XDS data analysis indicate that the capillary fluctuations at the two interfaces of the wetting films are partially correlated and their coupling is consistent with the van der Waals interactions. The relatively large intrinsic width $(4 \sim 6 \AA)$ of the liquid-liquid interface observed for thicker films $(D \gtrsim 50 \AA)$ is comparable to the value expected for the bulk liquid-liquid interface $(D \rightarrow \infty)$, determined by either the radius of gyration $(5.3 \AA)$ or the bulk correlation length $(4.8 \AA)$ of the alkane C20. The intrinsic liquid-vapor interfacial width is sharper $(\sim 2 \AA)$ and remains essentially constant over the entire probed range of $D$.
\end{abstract}

DOI: 10.1103/PhysRevE.74.031607 PACS number(s): 68.15.+e, 61.10.Kw, 68.05. $-\mathrm{n}, 68.03 .-\mathrm{g}$

\section{INTRODUCTION}

$\mathrm{X}$-ray and neutron reflectivity is the principal method for nondestructive characterization of the structure buried within nanometers of surfaces [1-4]. In addition to individual layer densities, thicknesses, and interfacial roughness, the reflectivity techniques can be used to extract more subtle structural features that may be technologically relevant. In particular, as demonstrated by extensive studies of solid multilayer films, the simultaneous measurements of x-ray specular reflectivity (XR) and off-specular diffuse scattering (XDS) provide a powerful tool for probing the layer-to-layer correlations in the roughness of neighboring interfaces [5-9]. The combination of XR and XDS has also been used to study the nature of roughness and/or the correlated roughness of multiple interfaces in substrate-supported thin films of liquids [10-12], polymers [13-16], and organic Langmuir-Blodgett multilayers [17-20].

For thin liquid films without solid substrates, the elucidation of correlated roughness, or equivalently film thickness fluctuations, is of fundamental interest since these effects reflect the form of the interfacial potential [21-23]. For example, in the first study of its kind for liquid films, Daillant et al. $[21,24]$ measured and theoretically analyzed the XDS from free-standing soap films and estimated the strength of

\footnotetext{
*Electronic address: fukuto@bnl.gov

†Electronic address: pershan@deas.harvard.edu
}

the coupling between the capillary fluctuations of the two (equivalent) liquid-vapor interfaces. More recently, Schlossman et al. $[22,25,26]$ carried out a combined XR/XDS study on an incomplete wetting film on bulk liquid and demonstrated the presence of partially correlated (i.e., neither independent nor perfectly conformal) capillary fluctuations at the liquid-vapor and liquid-liquid interfaces; furthermore, they used the extracted coupling constant to estimate the parameters for the short- and long-range interaction terms in the interfacial excess free energy [22].

Despite these capabilities, however, the application of $\mathrm{XR} / \mathrm{XDS}$ to purely fluid (or fluidlike) systems with multiple interfaces has been very limited [21-23,27]. This can be attributed partly to the experimental difficulties associated with liquid surfaces and films (flatness, stability, etc.). Another likely reason is that due to the subtleties of liquid surface scattering, proper interpretation of the data is nontrivial and tends to be technically involved. Thermal diffuse scattering from capillary fluctuations exhibits an algebraic singularity at the specular direction [28-30], and this causes the measured XR to depend on the experimental resolution [29-34]. For layered surfaces, not only is it more difficult to distinguish the capillary roughness at individual interfaces from non-step-like intrinsic interfacial profiles, but also the scattering is further complicated by its dependence on the partial correlations between the fluctuations of different interfaces. While the few published XR/XDS studies [21,22,27] have adequately dealt with some of these complications, not all of these technical issues have been explicitly addressed. For 
example, the data analysis in these previous studies follows the same general approach as used for solid surfaces that is based on the separation of scattering into the specular and diffuse contributions [28]. This approach is questionable given that the two contributions are experimentally indistinguishable for liquid surfaces [29,30]. Moreover, it appears that the effect of partially correlated roughness is usually neglected in the analysis of XR data and that the intrinsic widths of multiple interfaces are either too small to be extracted reliably or assumed to be zero.

We present here an XR/XDS study of the coupled thermal capillary fluctuations and the intrinsic profiles in complete wetting films adsorbed on the surface of bulk liquid. The purpose of this paper is twofold. The first objective is to present approximate methods for describing the surface $\mathrm{x}$-ray scattering from two fluctuating fluid-fluid interfaces and to demonstrate their accuracy and utility for data analysis. Our approach follows the well-known (and well-practiced) hybrid model of fluid surfaces $[35,36]$ in which each interface is assumed to have an intrinsic width and the thermally excited capillary waves are described in terms of macroscopic (i.e., lateral wave-vector-independent) surface tension and an upper wave-vector cutoff $[29,31,33,34,37-39]$. On the basis of these assumptions and the same Hamiltonian that Schlossman et al. used for the coupled capillary fluctuations [22], approximate behaviors of the lateral correlation functions for interfacial height and film thickness fluctuations are obtained. These approximate correlation functions are used to calculate, in the kinematic (Born) limit, normalized scattering intensities that can be compared with the data. In particular, rather than making an artificial distinction between the specular and diffuse contributions, the theoretical XR and XDS intensities are obtained by integrating one common expression for the differential cross section over realistic resolution functions. In addition, an approximation of the above integrals by Debye-Waller factors is used to derive a simple analytic form of specular reflectivity that takes into account the partial correlations between capillary fluctuations at the two interfaces.

The second objective is to present the results of XR/XDS measurements on wetting films of perfluoromethylcyclohexane $\left(\mathrm{PFMC}, \mathrm{C}_{6} \mathrm{~F}_{11}-\mathrm{CH}_{3}\right)$ on eicosane $\left[\mathrm{C} 20 ; \mathrm{CH}_{3}-\left(\mathrm{CH}_{2}\right)_{18}\right.$ $-\mathrm{CH}_{3}$ ]. Our initial XR study, described in Ref. [40], demonstrated the complete wetting of the bulk C20 surface by PFMC vapor. The present paper focuses on characterization of the coupled capillary fluctuations and the intrinsic interfacial widths at the liquid-liquid and liquid-vapor interfaces, over a range of film thickness $D(=30-160 \AA)$. PFMC and $\mathrm{C} 20$ are nonpolar, immiscible liquids and their intermolecular interactions are dominated by the long-range van der Waals (vdW) forces; therefore, PFMC wetting films on C20 should provide a simple model system for testing the expected coupling of capillary fluctuations at the two interfaces. The analysis of XR/XDS measurements indicate that (i) the capillary fluctuations at the two interfaces are consistent with the presence of interfacial coupling that is dominated by (nonretarded) vdW interactions, (ii) the intrinsic liquid-liquid interfacial width is in the range of 4-6 $\AA$ for $D \gtrsim 50 \AA$, comparable to both the radius of gyration $R_{g}$ $\approx 5.3 \AA$ for the $\mathrm{C} 20$ molecule [34] and an estimated bulk
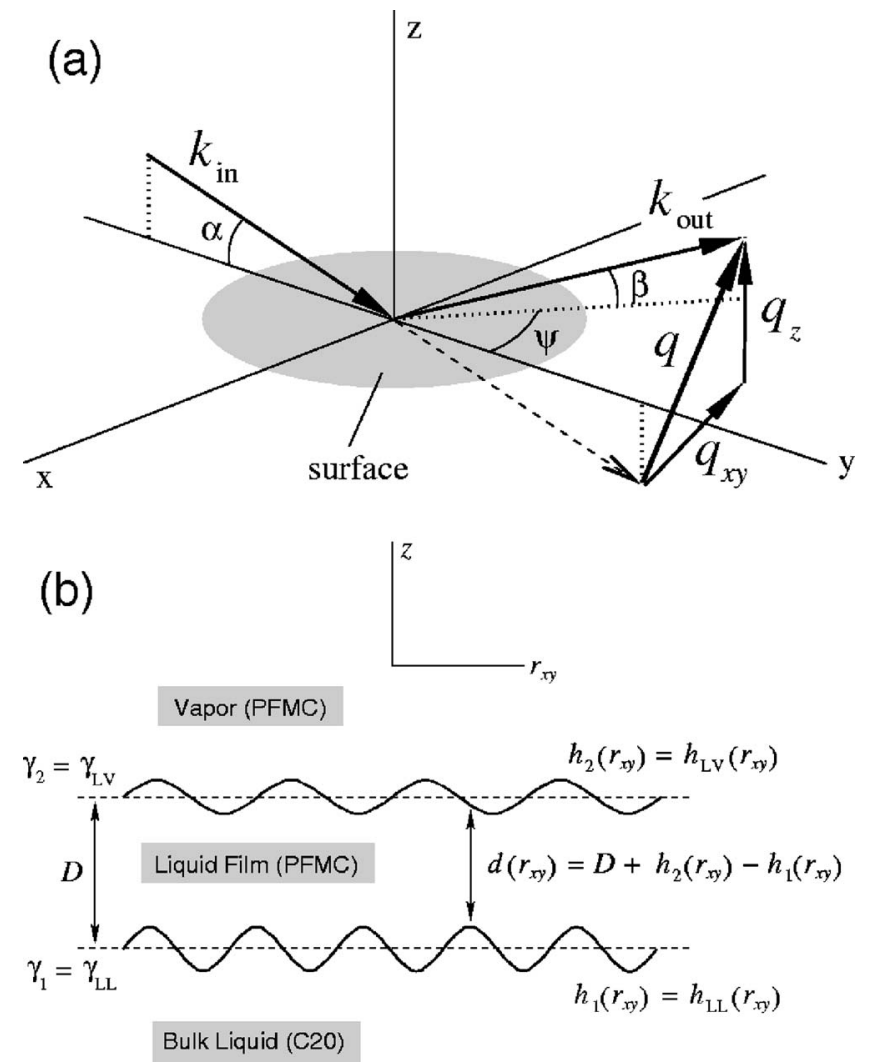

FIG. 1. (a) X-ray scattering geometry. (b) Illustration of a wetting film of average thickness $D$ on the surface of a bulk liquid. The liquid-liquid and liquid-vapor interfaces are denoted with indices 1 and 2, respectively. The local fluctuation in thickness is equal to the difference in the height fluctuations of the two interfaces at the same lateral position.

correlation length $\xi_{b} \approx 4.8 \AA$ for molten long-chain alkanes [38], and (iii) the intrinsic liquid-vapor interfacial width is sharper $(\sim 2 \AA)$ and remains nearly constant over the entire probed range of $D$.

This paper is organized as follows. Section II provides a brief background on x-ray scattering in order to establish the notations and describe the general formalism used to estimate XR/XDS intensities. In Sec. III, the capillary wave fluctuations at the two fluid-fluid interfaces of the wetting film and their effects on $\mathrm{x}$-ray scattering are considered in some detail. Section IV provides a brief description of experimental details. In Sec. V, the experimental results and the analysis of XR and XDS measurements are discussed. In Sec. VI, the main conclusions are summarized. Appendix A briefly describes the main characteristics of liquid surface scattering in the single-interface limit, which form the basis for the approximations developed in Sec. III.

\section{GENERAL BACKGROUND}

\section{A. X-ray kinematics}

The kinematics for surface $x$-ray scattering is illustrated in Fig. 1(a). The average surface lies in the $x-y$ plane, and the plane of incidence is taken to be the $y-z$ plane. The incident 
and scattered wave vectors are denoted as $\mathbf{k}_{\text {in }}$ and $\mathbf{k}_{\text {out }}$, respectively, and the wave-vector transfer is given by $\mathbf{q}=\mathbf{k}_{\text {out }}$ $-\mathbf{k}_{\text {in }}$. In terms of the incident angle $\alpha$ and the output angles $\beta$ and $\psi$ relative to the surface and the incidence plane, respectively, the components of $\mathbf{q}$ are given by

$$
\begin{gathered}
q_{z}=k[\sin (\alpha)+\sin (\beta)], \\
q_{x}=k \cos (\beta) \sin (\psi), \\
q_{y}=k[\cos (\beta) \cos (\psi)-\cos (\alpha)],
\end{gathered}
$$

where $k=2 \pi / \lambda$ and $\lambda$ is the $\mathrm{X}$-ray wavelength. The specular X-ray reflectivity (XR) is the fraction $R\left(q_{z}\right)=I\left(q_{z}\right) / I_{0}$ of the incident intensity $I_{0}$ that is reflected in the specular direction, i.e., at $\beta=\alpha$ and $\psi=0$ (or equivalently, $q_{x y}=0$ ), and it is measured as a function of $\alpha$ or $q_{z}=2 k \sin (\alpha)$. The diffuse scattering (XDS) from a surface is measured at nonspecular conditions $\beta \neq \alpha$, typically within the incidence plane $(\psi=0)$. For $\psi \approx 0$, the full width at half-maximum (FWHM) detector resolutions in terms of wave vectors and scattering angles are related to each other as $\Delta q_{x} \approx k \Delta \psi, \Delta q_{y} \approx k \sin (\beta) \Delta \beta$, and $\Delta q_{z} \approx k \Delta \beta$. In most XR and XDS experiments, the lateral wave-vector resolution is much wider transverse to the plane of incidence than along the incidence plane, i.e., $\Delta q_{x} \gg \Delta q_{y}$. Therefore, in typical measurements the smallest lateral wavevector that can be resolved is roughly of the order of $q_{x y, \min } \sim \Delta q_{y}$.

\section{B. X-ray scattering from a rough surface with two interfaces}

Figure 1(b) shows the convention used in this study to describe a two-interface surface, consisting of a film of average thickness $D$ on a subphase. The quantities referring to the film-subphase and film-vapor interfaces are denoted with subscript " 1 " and " 2 ," respectively. Deviations in the local interfacial heights from the average heights are represented by $\left\{h_{1}\left(\mathbf{r}_{x y}\right), h_{2}\left(\mathbf{r}_{x y}\right)\right\}$, where $\left\langle h_{i}(0)\right\rangle \equiv 0$.

$\mathrm{X}$-ray scattering from rough surfaces has been reviewed extensively $[4,24,28,30,41-45]$. The measured intensity $I\left(\mathbf{q}_{x y}, q_{z}\right)$ is proportional to the integration of the differential cross section $d \sigma / d \Omega$ over the solid angle $\Delta \Omega$ subtended by the detector slits. Equivalently, using the relation $\delta \Omega$ $=\cos (\beta) \delta \psi \delta \beta \approx \delta q_{x} \delta q_{y} /\left[k^{2} \sin (\beta)\right]$, the scattering intensity can be obtained by integration over the experimental resolutions in reciprocal space. For a film with two separate fluctuating interfaces, the normalized intensity can be expressed as

$$
\begin{aligned}
\frac{I\left(\mathbf{q}_{x y}, q_{z}\right)}{I_{0}}= & \frac{T_{F}(\alpha) T_{F}(\beta)}{4 q_{z}^{2} k^{2} \sin (\alpha) \sin (\beta)}\left(\frac{q_{c}}{2}\right)^{4} \\
& \times\left\{\sum_{i=1}^{2}\left|\Theta_{i}\left(q_{z}\right)\right|^{2} R_{i i}\left(\mathbf{q}_{x y}, q_{z}\right)\right. \\
& \left.+2 \Theta_{1}\left(q_{z}\right) \Theta_{2}\left(q_{z}\right) R_{12}\left(\mathbf{q}_{x y}, q_{z}\right) \cos \left(q_{z} D\right)\right\},
\end{aligned}
$$

where $q_{c}=4 \sqrt{\pi r_{0} \rho_{\infty}}=(4 \pi / \lambda) \sin \left(\alpha_{c}\right)$ is the critical wave vec- tor, $r_{0}$ is the classical radius of the electron, and $\rho_{\infty}$ is the electron density in the bulk subphase far below the surface [1]. Apart from the inclusion of the Fresnel transmission factors $T_{F}(\theta)$ to account for the surface enhancement effect near $\theta \sim \alpha_{c}$ [28,29], Eq. (2) is based on the kinematic (Born) approximation. For a simple case in which the interfacial profiles vary monotonically across each interface, the local (intrinsic) form factors for the two interfaces are commonly taken to be

$$
\begin{gathered}
\Theta_{1}\left(q_{z}\right)=\left(\phi_{1}-1\right) e^{-(1 / 2) w_{1}^{2} q_{z}^{2},} \\
\Theta_{2}\left(q_{z}\right)=-\phi_{1} e^{-(1 / 2) w_{2}^{2} q_{z}^{2},}
\end{gathered}
$$

where $w_{i}$ represents the intrinsic interfacial width and $\phi_{1}$ $=\rho_{1} / \rho_{\infty}$ is the electron density in the film relative to the bulk subphase. The dependence of observed intensity on both the interfacial roughnesses and the detector resolutions is contained in the factors

$$
\begin{aligned}
R_{i j}\left(\mathbf{q}_{x y}, q_{z}\right)= & \frac{1}{(2 \pi)^{2}} \int d^{2} \mathbf{q}_{x y}^{\prime} \Xi\left(\mathbf{q}_{x y}-\mathbf{q}_{x y}^{\prime}\right) \\
& \times \int_{A} d^{2} \mathbf{r}_{x y} e^{i \mathbf{q}_{x y}^{\prime} \cdot \mathbf{r}_{x y}} e^{-(1 / 2) g_{i j}\left(\mathbf{r}_{x y}\right) q_{z}^{2}},
\end{aligned}
$$

where $A$ is the surface area. The height-height correlation functions are defined as

$$
g_{i j}\left(\mathbf{r}_{x y}\right) \equiv\left\langle\left[h_{i}\left(\mathbf{r}_{x y}\right)-h_{j}(0)\right]^{2}\right\rangle .
$$

The resolution function $\Xi\left(\mathbf{q}_{x y}-\mathbf{q}_{x y}^{\prime}\right)$ in Eq. (4) satisfies $\Xi(0)=1$ at the detector center $\left(\mathbf{q}_{x y}^{\prime}=\mathbf{q}_{x y}\right)$ and $\Xi \rightarrow 0$ well outside the resolution widths. Note that for the most relevant case of scattering within the plane of incidence $(\psi=0)$, $q_{z}$ implicitly depends on $q_{y}^{\prime}$ and varies as $\delta q_{z} \approx k \delta \beta$ $\approx-\delta q_{y}^{\prime} / \sin (\beta)$. As is appropriate for typical experiments, Eq. (2) assumes that either the facor $\Theta_{i}\left(q_{z}\right)$ varies slowly enough with $q_{z}$ or the resolution is sufficiently fine that the minute variation in $\Theta_{i}\left(q_{z}\right)$ over the resolution volume can be neglected.

A kinematic expression for the specular reflectivity $R\left(q_{z}\right)=I\left(0, q_{z}\right) / I_{0}$ is given by the evaluation of Eq. (2) at $\mathbf{q}_{x y}=0($ or $\beta=\alpha)$ with $T_{F}(\theta)=\left(2 \theta / \alpha_{c}\right)^{2} \sqrt{R_{F}(\theta)}$,

$$
\begin{aligned}
\frac{R\left(q_{z}\right)}{R_{F}\left(q_{z}\right)}= & \sum_{i=1}^{2}\left|\Theta_{i}\left(q_{z}\right)\right|^{2} R_{i i}\left(0, q_{z}\right) \\
& +2 \Theta_{1}\left(q_{z}\right) \Theta_{2}\left(q_{z}\right) R_{12}\left(0, q_{z}\right) \cos \left(q_{z} D\right) .
\end{aligned}
$$

The Fresnel reflectivity $R_{F}\left(q_{z}\right)$ corresponds to an ideally flat and sharp surface for which the electron density changes discontinuously from the bulk value $\rho_{\infty}$ to zero [1]. $R_{F}\left(q_{z}\right)$ is equal to unity for $q_{z} \leqslant q_{c}$ (total external reflection) and has the form

$$
R_{F}\left(q_{z}\right) \approx\left(q_{c} / 2 q_{z}\right)^{4}
$$

for $q_{z} \gg q_{c}$.

The main objectives of XR/XDS experiments are to obtain measures of interfacial height fluctuations from the resolution-dependent roughness factors $R_{i j}\left(\mathbf{q}_{x y}, q_{z}\right)$ and to ex- 
tract the intrinsic profiles from the form factors $\Theta_{i}\left(q_{z}\right)$. These two types of effects can be separated if the nature of height fluctuations is known and the factors $R_{i j}\left(\mathbf{q}_{x y}, q_{z}\right)$ can be theoretically accounted for. This is done in the following way. By taking the Fourier transform

$$
\tilde{h}_{i}\left(\mathbf{q}_{x y}\right)=\int d^{2} \mathbf{r}_{x y} e^{i \mathbf{q}_{x y} \cdot \mathbf{x}_{x y}} h_{i}\left(\mathbf{r}_{x y}\right),
$$

the three real-space height-height correlation functions to be evaluated are

$$
\begin{aligned}
\frac{1}{2} g_{i i}\left(r_{x y}\right) & =\left\langle h_{i}^{2}(0)\right\rangle-\left\langle h_{i}\left(r_{x y}\right) h_{i}(0)\right\rangle \\
& =\frac{1}{2 \pi} \int_{0}^{q_{\max }} d q_{x y} q_{x y}\left[1-J_{0}\left(q_{x y} r_{x y}\right)\right] \frac{1}{A}\left\langle\left|\widetilde{h}_{i}\left(q_{x y}\right)\right|^{2}\right\rangle
\end{aligned}
$$

and

$$
g_{12}\left(r_{x y}\right)=\frac{1}{2}\left[g_{11}\left(r_{x y}\right)+g_{22}\left(r_{x y}\right)\right]+\left\langle\delta D\left(r_{x y}\right) \delta D(0)\right\rangle,
$$

where the thickness-thickness correlation function is given by

$$
\begin{aligned}
\left\langle\delta D\left(r_{x y}\right) \delta D(0)\right\rangle= & \left\langle\left[h_{2}\left(r_{x y}\right)-h_{1}\left(r_{x y}\right)\right]\left[h_{2}(0)-h_{1}(0)\right]\right\rangle \\
= & \frac{1}{2 \pi} \int_{0}^{q_{\max }} d q_{x y} q_{x y} J_{0}\left(q_{x y} r_{x y}\right) \\
& \times \frac{1}{A}\left\langle\left|\widetilde{h}_{2}\left(q_{x y}\right)-\widetilde{h}_{1}\left(q_{x y}\right)\right|^{2}\right\rangle .
\end{aligned}
$$

In Eqs. (9)-(11), the surface is assumed to be laterally isotropic and $r_{x y}=\left|\mathbf{r}_{x y}\right|$. The upper cutoff wave vector $q_{\max }$ corresponds to the smallest wavelength of the allowed height fluctuation modes, which is usually limited by the molecular size $a$, i.e., $q_{\max } \sim \pi / a[29,34,36]$.

Once $g_{i j}\left(r_{x y}\right)$ are known, the factors $R_{i j}\left(\mathbf{q}_{x y}, q_{z}\right)$ in Eq. (4) can be obtained, for example, by numerical integration using the slit-determined resolutions $\left(\Delta q_{x}, \Delta q_{y}\right)$ [46-51]. On the other hand, as demonstrated previously (see, e.g., Refs. $[15,21,24,29,52,53])$, the integration can be simplified considerably for scans at $\psi=0$ and small $\beta$ (i.e., for $\Delta q_{y} \ll \Delta q_{x}$ ) if the $\mathbf{q}_{x y}$ resolution function is taken to be infinitely wide in the $q_{x}$ direction. Substituting $\Xi\left(\mathbf{q}_{x y}-\mathbf{q}_{x y}^{\prime}\right)=\Xi\left(q_{y}-q_{y}^{\prime}\right)$ and taking the Fourier transform of the resolution function

$$
\Xi\left(q_{y}\right)=\int d y^{\prime} e^{i q_{y} y^{\prime}} \tilde{\Xi}\left(y^{\prime}\right),
$$

the integration of Eq. (4) over $\mathbf{q}_{x y}^{\prime}$ yields

$$
R_{i j}\left(q_{y}, q_{z}\right)=\int d y e^{i q_{y} y} e^{-(1 / 2) g_{i j}(y) q_{z}^{2}} \widetilde{\Xi}(y) .
$$

Since our measurements satisfy the condition $\Delta q_{y} \ll \Delta q_{x}$, this simplified form is used to evaluate $R_{i j}$ in Sec. III.

\section{LIQUID SURFACE WITH TWO INTERFACES}

Appendix A briefly describes (i) the peculiarities of thermal diffuse scattering arising from the capillary waves on liquid surfaces and (ii) approximations that have been used successfully to account for measured intensities for simple surfaces, i.e., those with either a single interface or conformal roughness. The aim of the present section is to develop similar approximations for surfaces in which there are two interacting fluid-fluid interfaces and their fluctuations are partially correlated with each other.

\section{A. Wetting and interfacial fluctuations}

The experimental surface to be discussed consists of a complete wetting film of PFMC on the surface of bulk liquid C20 [40]. The two fluctuating interfaces are illustrated in Fig. 1(b). The equilibrium thickness $D$ of a complete wetting film depends on the balance between (i) the energy cost of forming a liquid layer where the vapor is the stable bulk phase and (ii) the van der Waals (vdW) attractions between the wetting and subphase molecules. Taking $\Delta \mu$ to be the intrinsic chemical potential of the wetting molecules relative to the bulk liquid-vapor coexistence, $n$ to be the number density of the wetting liquid, and $A_{\text {eff }}$ to be the effective Hamaker constant for the planar bulk-film-vapor system, the thickness-dependent part of the surface excess free energy (per area) is given by $[54,55]$

$$
F(D)=\frac{A_{\text {eff }}}{12 \pi D^{2}}+n \Delta \mu D .
$$

The average film thickness $D$ is determined by minimization of Eq. (14),

$$
D=\left(\frac{A_{\text {eff }}}{6 \pi n \Delta \mu}\right)^{1 / 3} .
$$

The $1 / 3$ power-law dependence of $D$ on $\Delta \mu$ signifies complete wetting [56,57].

Following Li et al. [22], the energy cost for the interfacial height fluctuations $\left\{h_{1}\left(\mathbf{r}_{x y}\right), h_{2}\left(\mathbf{r}_{x y}\right)\right\}$ can be taken to be

$$
\begin{aligned}
U= & \int_{A} d^{2} \mathbf{r}_{x y}\left\{\frac{1}{2} \sum_{i=1}^{2}\left[\gamma_{i}\left|\nabla h_{i}\left(\mathbf{r}_{x y}\right)\right|^{2}+\Delta \rho_{m, i} g h_{i}^{2}\left(\mathbf{r}_{x y}\right)\right]\right. \\
& \left.+\frac{1}{2} B(D)\left[h_{2}\left(\mathbf{r}_{x y}\right)-h_{1}\left(\mathbf{r}_{x y}\right)\right]^{2}\right\},
\end{aligned}
$$

where $\left\{\gamma_{1}, \gamma_{2}\right\}$ are the surface tensions of the two interfaces, $B(D)$ is a thickness-dependent coupling constant between the capillary waves at the two interfaces, and $\Delta \rho_{m, i}$ refers to the mass density difference across each interface. In addition to the usual $\gamma_{i}$-dependent gradient terms and the gravitational terms for the individual interfaces, the $\mathrm{vdW}$ coupling term $[\propto B(D)]$ is included to account for the work required to change the local thickness from the average equilibrium value $D$. If the amplitudes of the fluctuations are small compared to $D$, this term can be taken to be the leading term in the power series expansion of $F(D+\delta D)-F(D)$ [via Eq. (14)] with $\delta D=h_{2}\left(\mathbf{r}_{x y}\right)-h_{1}\left(\mathbf{r}_{x y}\right)$, i.e., 


$$
B(D)=\frac{\partial^{2} F(D)}{\partial D^{2}}=\frac{A_{\mathrm{eff}}}{2 \pi D^{4}} .
$$

The fact that the coupling term is minimized if $h_{2}\left(\mathbf{r}_{x y}\right)$ $=h_{1}\left(\mathbf{r}_{x y}\right)$ implies that in the limit of small thickness or strong vdW attractions between the film and the subphase $[B(D)$ $\rightarrow \infty]$, the fluctuations of the two interfaces are primarily conformal. In the opposite limit of large $D$ or small $A_{\text {eff }}[B(D) \rightarrow 0]$, Eq. (16) reduces to the sum of Hamiltonians at the two individual interfaces, and therefore the two interfaces should fluctuate independently, as expected for macroscopically thick films.

The above treatment of the coupling term is implicitly based on the Derjaguin approximation (DA) [58-60]. In the present context, the DA implies that (i) the local energy density (per unit area) at a given point $\mathbf{r}_{x y}$ for the interactions between the two fluctuating interfaces is assumed to depend only on the local thickness $d\left(\mathbf{r}_{x y}\right)=D+h_{2}\left(\mathbf{r}_{x y}\right)-h_{1}\left(\mathbf{r}_{x y}\right)$, and (ii) this local energy density is approximated with the value that would be obtained for a pair of flat and parallel interfaces that are separated by $d\left(\mathbf{r}_{x y}\right)$. As shown by Andelman et al. $[59,60]$ for wetting films on rough solid surfaces, the DA should be valid for $q_{x y} \ll 1 / D$, i.e., for describing the coupling between interfacial fluctuations of long wavelengths, but is expected to break down for $q_{x y} \gg 1 / D$. This limitation of the DA is not a serious problem here since the $\mathrm{x}$-ray scattering measurements in the present study were taken at relatively small magnitudes of the lateral wave vectors $\quad\left[\left|q_{y}\right| \leqslant \max \left\{\left|q_{y}\right|\right\} \sim 2 \times 10^{-3} \AA^{-1}<1 / \max \{D\} \sim 6\right.$ $\left.\times 10^{-3} \AA^{-1}\right]$ and should thus be dominated by the longwavelength behavior.

Using the Fourier transform of $h_{i}\left(\mathbf{r}_{x y}\right)$ in Eq. (8), the equipartition of the Fourier modes in the Hamiltonian [Eq. (16)] yields the mean-square amplitudes of height and thickness fluctuations,

$$
\begin{aligned}
& \frac{1}{A}\left\langle\widetilde{h}_{i}^{*}\left(q_{x y}\right) \widetilde{h}_{j}\left(q_{x y}\right)\right\rangle \cong \delta_{i j} \frac{k_{B} T}{\gamma_{i}} \frac{1}{q_{x y}^{2}+\xi^{-2}} \\
&+\frac{k_{B} T}{\gamma_{1}+\gamma_{2}}\left[\frac{1}{q_{x y}^{2}+\xi_{g}^{2}}-\frac{1}{q_{x y}^{2}+\xi^{-2}}\right], \\
& \frac{1}{A}\left\langle\left|\widetilde{h}_{2}\left(q_{x y}\right)-\widetilde{h}_{1}\left(q_{x y}\right)\right|^{2}\right\rangle \cong \frac{k_{B} T\left(\gamma_{1}+\gamma_{2}\right)}{\gamma_{1} \gamma_{2}} \frac{1}{q_{x y}^{2}+\xi^{-2}},
\end{aligned}
$$

where $\delta_{i j}$ is the Kronecker delta. The presence of two interfaces leads to two lateral correlation lengths,

$$
\begin{gathered}
\xi^{2}=\frac{1}{B(D)} \frac{\gamma_{1} \gamma_{2}}{\gamma_{1}+\gamma_{2}}=\frac{2 \pi D^{4}}{A_{\text {eff }}} \frac{\gamma_{1} \gamma_{2}}{\gamma_{1}+\gamma_{2}}, \\
\xi_{g}^{2}=\frac{\gamma_{1}+\gamma_{2}}{\left(\Delta \rho_{m, 1}+\Delta \rho_{m, 2}\right) g}=\frac{\gamma_{1}+\gamma_{2}}{\rho_{m, \infty} g},
\end{gathered}
$$

where $\rho_{m, \infty}=\Delta \rho_{m, 1}+\Delta \rho_{m, 2}$ is equal to the mass density of the bulk liquid. The vdW-limited correlation length $\xi \sim D^{2}$ is a measure of how far the correlations in thickness fluctuations persist over the surface [see Eq. (19)]; it ranges from intermolecular length scales for very thin films to $\sim 1 \mu \mathrm{m}$ for films that are a few hundred angstroms thick. As in the case of simple liquid surfaces (Appendix A), the gravity-limited length $\xi_{g}$ is of the order of $\sim 1 \mathrm{~mm}$ and sets the correlation length for the height fluctuations.

It is clear from the above that the two types of height fluctuations contribute to $\left\langle\left|\widetilde{h}_{i}\left(q_{x y}\right)\right|^{2}\right\rangle$. For each interface $(i$ $=j$ ), the first term in Eq. (18), which depends only on the surface tension $\gamma_{i}$ of that interface and has the same form as for the simple liquid-vapor interface [31,36], describes the component of independent fluctuations. The second term in Eq. (18), which is exactly the same for all $\{i, j\}$ and depends on the effective surface tension $\gamma_{\mathrm{eff}}=\gamma_{1}+\gamma_{2}$, represents the component of conformal fluctuations. The mean-square amplitudes above will be used below to derive approximate expressions for the real-space correlation functions and to estimate the Debye-Waller-like effects on the reflectivity.

\section{B. Approximate correlation functions}

For simple liquid surfaces (Appendix A), it has proven practical to approximate the scattering cross section by extending the logarithmic behavior of $g\left(r_{x y}\right)$ for $q_{\max }^{-1} \ll r_{x y}$ $\ll \xi_{g}$ [Eq. (A3)] to the entire range $r_{x y} \geqslant q_{\max }^{-1}$. An equivalent approximation for two fluctuating interfaces can be developed by considering the limiting behavior of the correlation functions in Eqs. (9)-(11). On the basis of Eqs. (18) and (19), the correlation functions behave approximately as follows [61]:

$$
\begin{aligned}
\left\langle h_{i}\left(r_{x y}\right) h_{j}(0)\right\rangle \approx & \frac{k_{B} T}{2 \pi}\left[\frac{1}{\gamma_{1}+\gamma_{2}} \ln \left(\frac{\xi_{g}}{\xi}\right)\right. \\
& \left.+\delta_{i j} \frac{1}{\gamma_{i}} \ln \left(q_{\max } \xi\right)\right] \text { for } r_{x y}=0 \\
\approx & \frac{k_{B} T}{2 \pi}\left[\frac{1}{\gamma_{1}+\gamma_{2}} \ln \left(\frac{\xi_{g}}{\xi}\right)\right. \\
& \left.+\delta_{i j} \frac{1}{\gamma_{i}} \ln \left(\frac{\xi}{r_{x y}}\right)\right] \text { for } q_{\max }^{-1} \ll r_{x y} \ll \xi \\
\approx & \frac{k_{B} T}{2 \pi} \frac{1}{\gamma_{1}+\gamma_{2}} \ln \left(\frac{\xi_{g}}{r_{x y}}\right) \text { for } \xi \ll r_{x y} \ll \xi_{g} \\
\approx & 0 \text { for } r_{x y} \gg \xi_{g}, \\
\frac{1}{2} g_{i i}\left(r_{x y}\right)= & 0 \text { for } r_{x y}=0(\operatorname{exact}) \\
\approx & \frac{k_{B} T}{2 \pi} \frac{1}{\gamma_{i}} \ln \left(q_{\max } r_{x y}\right) \text { for } q_{\max }^{-1} \ll r_{x y} \ll \xi \\
\approx & \frac{k_{B} T}{2 \pi}\left[\frac{1}{\gamma_{i}} \ln \left(q_{\max } \xi\right)\right. \\
& \left.+\frac{1}{\gamma_{1}+\gamma_{2}} \ln \left(\frac{r_{x y}}{\xi}\right)\right] \text { for } \xi \ll r_{x y} \ll \xi_{g} \\
\approx & \frac{k_{B} T}{2 \pi}\left[\frac{1}{\gamma_{i}} \ln \left(q_{\max } \xi\right)\right. \\
+ & \left.\frac{1}{\gamma_{1}+\gamma_{2}} \ln \left(\frac{\xi_{g}}{\xi}\right)\right] \text { for } r_{x y} \gg \xi_{g}, \\
\text { fing } &
\end{aligned}
$$




$$
\begin{aligned}
\frac{1}{2} g_{12}\left(r_{x y}\right) \approx & \frac{k_{B} T}{2 \pi} \frac{1}{2}\left(\frac{1}{\gamma_{1}}+\frac{1}{\gamma_{2}}\right) \ln \left(q_{\max } \xi\right) \text { for } r_{x y} \ll \xi \\
\approx & \frac{k_{B} T}{2 \pi}\left[\frac{1}{2}\left(\frac{1}{\gamma_{1}}+\frac{1}{\gamma_{2}}\right) \ln \left(q_{\max } \xi\right)\right. \\
& \left.+\frac{1}{\gamma_{1}+\gamma_{2}} \ln \left(\frac{r_{x y}}{\xi}\right)\right] \text { for } \xi \ll r_{x y} \ll \xi_{g} \\
\approx & \frac{k_{B} T}{2 \pi}\left[\frac{1}{2}\left(\frac{1}{\gamma_{1}}+\frac{1}{\gamma_{2}}\right) \ln \left(q_{\max } \xi\right)\right. \\
& \left.+\frac{1}{\gamma_{1}+\gamma_{2}} \ln \left(\frac{\xi_{g}}{\xi}\right)\right] \text { for } r_{x y} \gg \xi_{g}, \\
\left\langle\delta D\left(r_{x y}\right) \delta D(0)\right\rangle \approx & \frac{k_{B} T}{2 \pi}\left(\frac{1}{\gamma_{1}}+\frac{1}{\gamma_{2}}\right) \ln \left(q_{\max } \xi\right) \text { for } r_{x y}=0 \\
\approx & \frac{k_{B} T}{2 \pi}\left(\frac{1}{\gamma_{1}}+\frac{1}{\gamma_{2}}\right) \ln \left(\frac{\xi}{r_{x y}}\right) \text { for } q_{\max }^{-1} \ll r_{x y} \ll \xi \\
\approx & 0 \text { for } r_{x y} \gg \xi .
\end{aligned}
$$

For each correlation function, note that the limiting forms in adjacent ranges of $r_{x y}$ are continuous and extrapolate to identical values at $r_{x y}=\xi$ and at $r_{x y}=\xi_{g}$, and the substitution of $r_{x y}=q_{\max }^{-1}$ in the expression for $r_{x y} \gg q_{\max }^{-1}$ results in the mean-square value at $r_{x y}=0$. These limiting forms are plotted in Figs. 2(a) and 2(b), for a PFMC film on C20 at $42{ }^{\circ} \mathrm{C}$ (see Table I) and $D=100 \AA$.

The essential properties of the correlation functions are captured by the above limiting forms, including the basic features of $\left\langle h_{i}\left(r_{x y}\right) h_{j}(0)\right\rangle$ that have been discussed previously by Daillant et al. [24]. In addition, it is shown explicitly that $\xi$ sets the lateral correlation length for thickness fluctuations $\left[\left\langle\delta D\left(r_{x y}\right) \delta D(0)\right\rangle\right.$, Fig. 2(b)] and $\xi_{g}$ sets the correlation length for height fluctuations $\left[\left\langle h_{i}\left(r_{x y}\right) h_{j}(0)\right\rangle\right.$, Fig. 2(a)]. The correlation functions always display logarithmic dependence on $r_{x y}$ over the ranges of $r_{x y}$ where they vary; however, the behavior of each correlation function is different above and below $\xi$. For $r_{x y} \ll \xi$, in addition to the common constant term in all three functions $\left\langle h_{i}\left(r_{x y}\right) h_{j}(0)\right\rangle$ [Eq. (22) and Fig. 2(a)], each self-correlation function $(i=j)$ contains a logarithmic term that depends only on the surface tension $\gamma_{i}$ of the relevant interface. Therefore, both the independent and conformal fluctuations contribute to the self-correlations $\left\langle h_{i}\left(r_{x y}\right) h_{i}(0)\right\rangle$ $\neq 0$, whereas the cross-correlations $\left\langle h_{1}\left(r_{x y}\right) h_{2}(0)\right\rangle \neq 0$ originate solely from the conformal part. The presence of independent height fluctuations is reflected in the different growth rates of $g_{11}\left(r_{x y}\right)$ and $g_{22}\left(r_{x y}\right)$ for $r_{x y}<\xi$ and in the $r_{x y}$ dependence of the nonconformal thickness fluctuations $\left\langle\delta D\left(r_{x y}\right) \delta D(0)\right\rangle \neq 0$. For $\xi \ll r_{x y} \ll \xi_{g}$, the fluctuations are completely conformal $\left[\left\langle\delta D\left(r_{x y}\right) \delta D(0)\right\rangle=0\right]$; all three functions $\left\langle h_{i}\left(r_{x y}\right) h_{j}(0)\right\rangle$ become identical and follow a common logarithmic falloff with $r_{x y}$ that depends on the effective surface tension $\gamma_{\mathrm{eff}}=\gamma_{1}+\gamma_{2}$. For $r_{x y} \gg \xi_{g}$, the height fluctuations become uncorrelated, which necessarily implies $\left\langle\delta D\left(r_{x y}\right) \delta D(0)\right\rangle=0$.
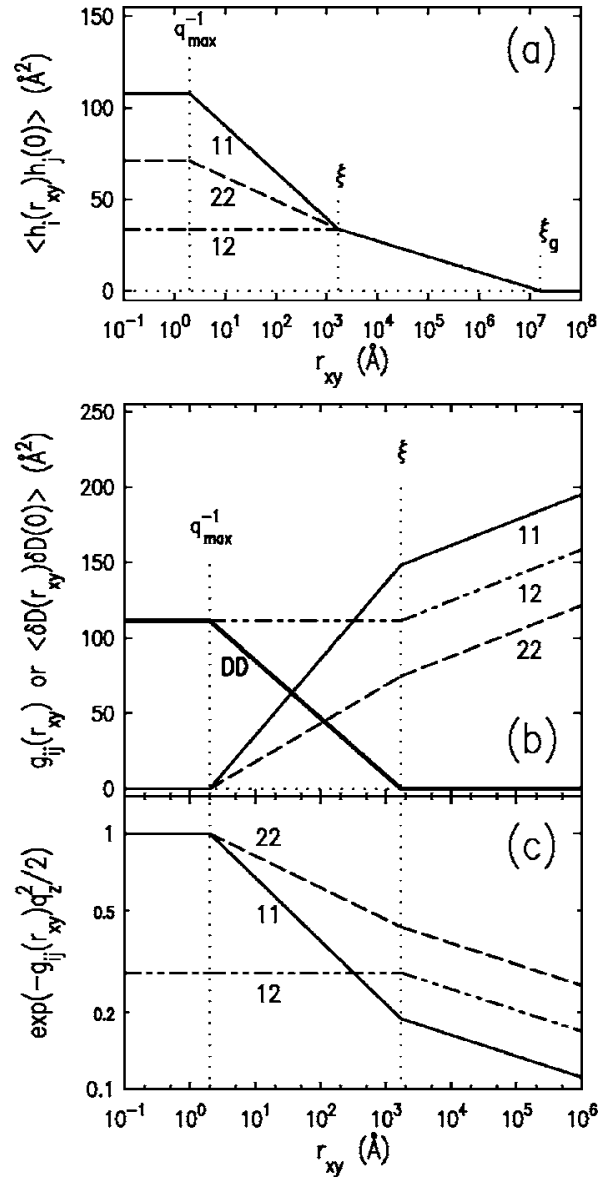

FIG. 2. Approximate forms of various correlation functions for the vdW-coupled capillary fluctuations at the liquid-liquid and liquid-vapor interfaces as a function of lateral separation $r_{x y}$. The curves are calculated using the parameters in Table I for a PFMC wetting film on bulk $\mathrm{C} 20$ at $42{ }^{\circ} \mathrm{C}$ and $D=100 \AA$, corresponding to $\xi=1700 \AA$ A. (a) $\left\langle h_{i}\left(r_{x y}\right) h_{j}(0)\right\rangle$ (labeled $i j$ ) from Eq. (22); (b) $g_{i j}\left(r_{x y}\right)$ (labeled $i j$ ) and $\left\langle\delta D\left(r_{x y}\right) \delta D(0)\right\rangle$ (labeled " $D D$ "), from Eqs. (23)-(25); (c) $\exp \left[-\frac{1}{2} g_{i j}\left(r_{x y}\right) q_{z}^{2}\right]$ at $q_{z}=0.15 \AA^{-1}$, from Eqs. (26) and (27).

For the purpose of estimating the scattered intensity [Eqs. (2), (4), and (6)], the correlation functions can be simplified further. First, for the typical film thickness probed here $(D$ $=30-160 \AA)$, the inequalities $q_{\max }^{-1}(\sim 2 \AA) \ll \xi \quad(=150$ $-4400 \AA) \ll \xi_{g}(\sim 1 \mathrm{~mm})$ are generally satisfied. Since these lengths differ significantly in magnitude and are independent of the precise form of correlation functions, a reasonable approximation is to neglect the gradual crossover behaviors near $r_{x y}=\left\{q_{\max }^{-1}, \xi, \xi_{g}\right\}$ and apply the limiting forms in Eqs. (22)-(25) to all values of $r_{x y}$ within each length scale region. Second, since it is impossible to resolve $\xi_{g}$ in practical x-ray experiments $\left(q_{x y, \min } \sim \Delta q_{y}=10^{-5}-10^{-4} \AA^{-1} \gg \xi_{g}^{1}\right)$, the limiting forms of $g_{i j}\left(r_{x y}\right)$ for $\xi \ll r_{x y} \ll \xi_{g}$, which are independent of $\xi_{g}$, will be extended to large $r_{x y} \rightarrow \infty$. This same approximation has been used for $g\left(r_{x y}\right)$ for simple surfaces (Appendix A). Applying these approximations to Eqs. (23) and (24), obtains 
TABLE I. Values of the parameters relevant to the present experiment.

\begin{tabular}{|c|c|}
\hline Quantity & Value \\
\hline Temperature & $\begin{array}{l}T_{1}=37.2{ }^{\circ} \mathrm{C} \\
T_{2}=42.2{ }^{\circ} \mathrm{C}\end{array}$ \\
\hline Effective Hamaker constant ${ }^{a}$ & $\begin{aligned} A_{\mathrm{eff}} & =A_{\mathrm{HF}}-A_{\mathrm{FF}} \\
& =0.9 \times 10^{-20} \mathrm{~J}\end{aligned}$ \\
\hline Liquid-liquid surface tension ${ }^{b}$ & $\begin{array}{l}\gamma_{1}=6.5 \mathrm{dyn} / \mathrm{cm} \text { at } T_{1} \\
\gamma_{1}=6.3 \mathrm{dyn} / \mathrm{cm} \text { at } T_{2}\end{array}$ \\
\hline Liquid-vapor surface tension $^{c}$ & $\gamma_{2}=12.5 \mathrm{dyn} / \mathrm{cm}$ \\
\hline 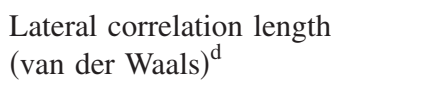 & $\xi=\left(0.17 \AA^{-1}\right) D^{2}$ \\
\hline 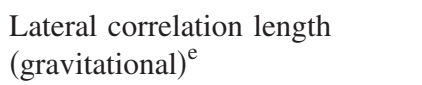 & $\xi_{g}=1.6 \mathrm{~mm}$ \\
\hline Upper wave-vector cutoff $^{f}$ & $q_{\max }=\pi / a=0.5 \AA^{-1}$ \\
\hline Relative layer electron density ${ }^{\mathrm{g}}$ & $\rho_{\mathrm{PFMC}} / \rho_{\mathrm{C} 20}=1.9$ \\
\hline
\end{tabular}

${ }^{\mathrm{a}}$ For fluorocarbon film on bulk hydrocarbon [66].

${ }^{\mathrm{b}}$ For bulk PFMC-C20 interface, via pendant drop method [65].

${ }^{c}$ For PFMC-vapor interface [66].

${ }^{\mathrm{d}}$ From Eq. (20).

${ }^{\mathrm{e}}$ From Eq. (21) and $\rho_{m, \infty}=0.79 \mathrm{~g} / \mathrm{cm}^{3}$ for $\mathrm{C} 20$.

${ }^{\mathrm{f}}$ From molecular diameter of $a \sim 6 \AA$ for PFMC [66].

${ }^{\mathrm{g}}$ Nominal value estimated from mass densities; treated as a fitting parameter for $R / R_{F}$.

$$
\begin{aligned}
e^{-(1 / 2) g_{i i}\left(r_{x y}\right) q_{z}^{2}} & \approx 1 \text { for } r_{x y} \leqslant q_{\max }^{-1} \\
& \approx\left(q_{\max } r_{x y}\right)^{-\eta_{i}} \text { for } q_{\max }^{-1}<r_{x y} \leqslant \xi \\
& \approx\left(q_{\max } \xi\right)^{-\eta_{i}}\left(r_{x y} / \xi\right)^{-\eta_{12}} \text { for } r_{x y}>\xi \\
e^{-(1 / 2) g_{12}\left(r_{x y}\right) q_{z}^{2}} & \approx\left(q_{\max } \xi\right)^{-(1 / 2)\left(\eta_{1}+\eta_{2}\right)} \text { for } r_{x y} \leqslant \xi \\
& \approx\left(q_{\max } \xi\right)^{-(1 / 2)\left(\eta_{1}+\eta_{2}\right)}\left(r_{x y} / \xi\right)^{-\eta_{12}} \text { for } r_{x y}>\xi
\end{aligned}
$$

where the $q_{z}$-dependent exponents are defined as

$$
\begin{gathered}
\eta_{i} \equiv \frac{k_{B} T}{2 \pi \gamma_{i}} q_{z}^{2}, \\
\eta_{12} \equiv \frac{k_{B} T}{2 \pi\left(\gamma_{1}+\gamma_{2}\right)} q_{z}^{2} .
\end{gathered}
$$

Figure 2(c) shows the behavior of these approximate functions over the range $r_{x y}<\xi_{g}$, calculated for $q_{z}=0.15 \AA^{-1}$ and the same parameters as in Figs. 2(a) and 2(b).

Using the above approximate forms of $\exp \left[-\frac{1}{2} g_{i j}\left(r_{x y}\right) q_{z}^{2}\right]$, the evaluation of $R_{i j}\left(q_{y}, q_{z}\right)$ is reduced to a single numerical integration of simple analytic functions in real space. This is illustrated below for XDS and XR separately, choosing a realistic resolution function that is appropriate for each technique.

\section{Off-specular diffuse scattering}

In order to maximize the dynamic range of the XDS data, it is often advantageous to have access to as small a lateral

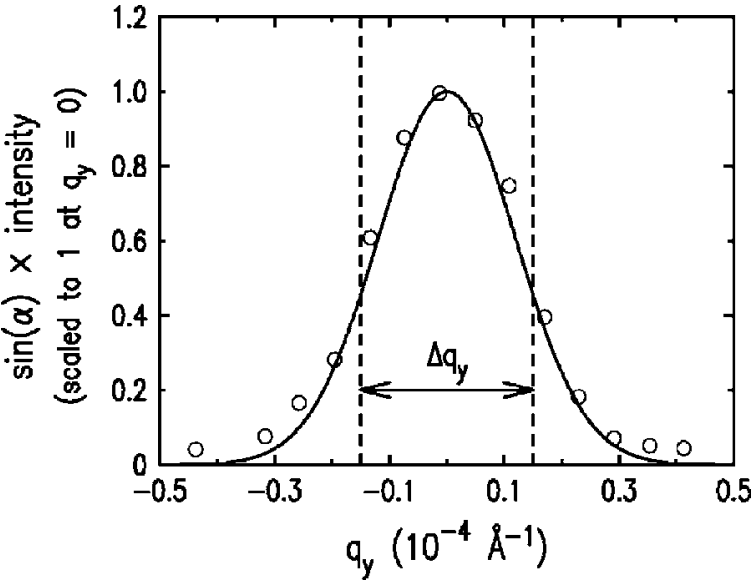

FIG. 3. An XDS rocking scan in the incidence plane $\left(q_{x}=0, \psi\right.$ $=0)$ at $q_{z}=0.14 \AA^{-1}$, taken with an experimental resolution of $\Delta q_{y}$ $\approx\left(q_{z} / 2\right) \Delta \beta=3.0 \times 10^{-5} \AA^{-1}$. The data $(\bigcirc)$ correspond to a PFMC wetting film on $\mathrm{C} 20$ at $42{ }^{\circ} \mathrm{C}$ and $D=155 \AA$ and is identical to the data in Fig. 7(c). The observed intensity is shown as the product $\sin (\alpha) I\left(q_{y}, q_{z}\right)$, which accounts for the change in the x-ray footprint on the surface with varying $q_{y}$ and should be symmetric with respect to the sign of $q_{y}$ for fixed $q_{z}$. The solid curve corresponds to the Gaussian resolution function $\Xi\left(q_{y}-q_{y}^{\prime}\right)$ in Eq. (29). For comparison, the data $\sin (\alpha) I\left(q_{y}, q_{z}\right)$ are normalized to be unity at $q_{y}$ $=0$.

wave vector $\left(q_{x y, \min } \sim \Delta q_{y}\right)$ as possible. Ultimately, the resolution is limited by either the angular divergence of the incident beam $\left(\Delta \alpha \sim 10^{-4}\right.$ rad for the measurements below) or the finite curvature of the liquid surface. The detector angular resolution $\Delta \beta$ for $\mathrm{XDS}$ should be chosen such that it is as close to this limit as possible and still wide enough to yield measurable intensities at higher $q_{z}$. The XDS measurements in the present study were taken with $\Delta \beta=4.3 \times 10^{-4} \mathrm{rad}$ (Sec. IV B); a typical rocking curve scan around the specular condition is shown in Fig. 3. It is clear that the FWHM of the central peak is close to the slit-based width $\Delta q_{y}=\left(q_{z} / 2\right) \Delta \beta$, and that the experimental resolution is well represented by a Gaussian $[15,29,52]$ of the form

$$
\Xi\left(q_{y}-q_{y}^{\prime}\right)=\exp \left[-\pi\left(q_{y}-q_{y}^{\prime}\right)^{2} /\left(\Delta q_{y}\right)^{2}\right] .
$$

This expression satisfies $\Xi(0)=1$ at the detector center, and the integration of $\Xi$ over $(-\infty, \infty)$ yields the FWHM resolution $\Delta q_{y}$.

Substituting the Fourier transform of Eq. (29) in Eq. (13),

$$
R_{i j}\left(q_{y}, q_{z}\right)=\frac{\Delta q_{y}}{\pi} \int_{0}^{\infty} d y \cos \left(q_{y} y\right) e^{-\left(\Delta q_{y} y\right)^{2} / 4 \pi} e^{-(1 / 2) g_{i j}(y) q_{z}^{2}}
$$

This integration can be carried out numerically using the approximate factors $\exp \left[-\frac{1}{2} g_{i j}\left(r_{x y}\right) q_{z}^{2}\right]$ in Eqs. (26) and (27). Due to the relatively fast decay of the integrand with $y$, the numerical integration converges without difficulties. The normalized intensity $I\left(q_{y}, q_{z}\right) / I_{0}$ to be compared with XDS data can be obtained using Eq. (2). 
In the limit of very weak $\left(\xi \rightarrow \xi_{g}\right.$ or $\left.\infty\right)$ or strong ( $\xi$ $\left.\rightarrow q_{\max }^{-1}\right)$ interfacial coupling, the factors $\exp \left[-\frac{1}{2} g_{i j}\left(r_{x y}\right) q_{z}^{2}\right]$ are simplified such that the integral in Eq. (30) can be solved analytically, in the manner similar to the case of simple liquid-vapor interfaces [29]. The limit of weak coupling or independent fluctuations yields

$$
\begin{aligned}
& \lim _{\xi \rightarrow \xi_{g} \rightarrow \infty} R_{i i}\left(q_{y}, q_{z}\right) \approx 2(4 \pi)^{-(1 / 2)\left(1+\eta_{i}\right)} \Gamma\left(\frac{1-\eta_{i}}{2}\right) \\
& \times\left(\frac{\Delta q_{y}}{q_{\max }}\right)^{\eta_{i}}{ }_{1} F_{1}\left(\frac{1-\eta_{i}}{2} ; \frac{1}{2} ;-\pi\left[\frac{q_{y}}{\Delta q_{y}}\right]^{2}\right) \\
& \approx \frac{1}{\pi} \Gamma\left(1-\eta_{i}\right) \sin \left(\frac{\pi \eta_{i}}{2}\right) \frac{\Delta q_{y}}{\left|q_{y}\right|}\left(\frac{\left|q_{y}\right|}{q_{\max }}\right)^{\eta_{i}} \\
& \text { for }\left|q_{y}\right| \gg \Delta q_{y} \text {, } \\
& \lim _{\xi \rightarrow \xi_{g}} R_{12}\left(q_{y}, q_{z}\right) \approx e^{-(1 / 2)\left[\left\langle h_{1}^{2}(0)\right\rangle+\left\langle h_{2}^{2}(0)\right\rangle\right] q_{z}^{2}} e^{-\pi\left(q_{y} / \Delta q_{y}\right)^{2}} \\
& \sim 0 \text { for }\left|q_{y}\right| \gg \Delta q_{y},
\end{aligned}
$$

where $\Gamma$ is the Gamma function and ${ }_{1} F_{1}$ is the Kumer function. The asymptotic behavior of $R_{i j}$ is consistent with the fact that the diffuse scattering from independently fluctuating interfaces contains no interference term. In the opposite limit of strong coupling or conformal fluctuations, the three $R_{i j}$ factors become identical,

$$
\begin{aligned}
\lim _{\xi \rightarrow q_{\max }^{-1}} R_{i j}\left(q_{y}, q_{z}\right) \approx & 2(4 \pi)^{-(1 / 2)\left(1+\eta_{12}\right)} \Gamma\left(\frac{1-\eta_{12}}{2}\right) \\
& \times\left(\frac{\Delta q_{y}}{q_{\max }}\right)^{\eta_{12}}{ }_{1} F_{1}\left(\frac{1-\eta_{12}}{2} ; \frac{1}{2} ;-\pi\left[\frac{q_{y}}{\Delta q_{y}}\right]^{2}\right) \\
\approx & \frac{1}{\pi} \Gamma\left(1-\eta_{12}\right) \sin \left(\frac{\pi \eta_{12}}{2}\right) \frac{\Delta q_{y}}{\left|q_{y}\right|}\left(\frac{\left|q_{y}\right|}{q_{\max }}\right)^{\eta_{12}} \\
& \text { for }\left|q_{y}\right| \gg \Delta q_{y} .
\end{aligned}
$$

For $\eta_{12} \ll 1$, the asymptotic form reduces to the singlesurface expression Eq. (A4) with $\Xi\left(\mathbf{q}_{x y}-\mathbf{q}_{x y}^{\prime}\right)=\Delta q_{y} \delta\left(q_{y}\right.$ $\left.-q_{y}^{\prime}\right)$ and $\eta=\eta_{12}$.

In Sec. V the measured XDS intensities will be compared with the predicted form based on Eq. (30) for the partially correlated interfaces as well as the above limiting cases.

\section{Specular reflectivity}

In general, XR from liquid surfaces is measured with a resolution that is sufficiently coarse as to integrate over the near-singular cusplike peak in the differential cross section at $\mathbf{q}_{x y}=0$ (Appendix A). In slits-based XR measurements, the resolution is usually closely approximated by the rectangular form

$$
\Xi\left(q_{y}-q_{y}^{\prime}\right)= \begin{cases}1 & \text { if }\left|q_{y}-q_{y}^{\prime}\right|<\frac{1}{2} \Delta q_{y} \\ 0 & \text { otherwise. }\end{cases}
$$

One method to calculate $R_{i j}\left(0, q_{z}\right)$ for XR [Eq. (6)] is to follow the same approach as for XDS. Substituting the Fou- rier transform of Eq. (34) in Eq. (13) and setting $q_{y}=0$,

$$
R_{i j}\left(0, q_{z}\right)=\frac{\Delta q_{y}}{\pi} \int_{0}^{\infty} d y \frac{\sin \left(\Delta q_{y} y / 2\right)}{\Delta q_{y} y / 2} e^{-(1 / 2) g_{i j}(y) q_{z}^{2}} .
$$

This integration can be carried out numerically using the approximate forms of $\exp \left[-\frac{1}{2} g_{i j}\left(r_{x y}\right) q_{z}^{2}\right]$ in Eqs. (26) and (27). While the numerical integration is straightforward to implement, this approach has two disadvantages. First, although the effects of various interfacial parameters on reflectivity can in principle be calculated, an intuitive understanding of these effects is difficult to gain using this approach. Second, both the fast oscillation and the slow decay of the integrand for some values of $y$ cause the numerical integration process to be relatively slow and inconvenient for use in the XR data analysis that involves iterative fitting.

The alternative approach that works well for small $q_{z}$ is to approximate the factors $R_{i j}\left(0, q_{z}\right)$ by Gaussians of DebyeWaller (DW) form,

$$
R_{i j}\left(0, q_{z}\right)=e^{-\sigma_{c, i j}^{2} q_{z}^{2}},
$$

where each capillary-wave roughness parameter $\sigma_{c, i j}$ depends on $g_{i j}\left(r_{x y}\right)$ and the resolution function. The simplest way to derive the expressions for $\sigma_{c, i j}$ is to expand $\exp \left[-\frac{1}{2} g_{i j}\left(r_{x y}\right) q_{z}^{2}\right]$ in Eq. (4) as a power series and identify $\sigma_{c, i j}^{2}$ with the coefficient for the $q_{z}^{2}$ term, leading to

$$
\begin{aligned}
\sigma_{c, i j}^{2}= & \frac{1}{2}\left[\left\langle h_{i}^{2}(0)\right\rangle+\left\langle h_{j}^{2}(0)\right\rangle\right] \\
& -\frac{1}{(2 \pi)^{2}} \int d^{2} \mathbf{q}_{x y}^{\prime} \Xi\left(\mathbf{q}_{x y}^{\prime}\right) \frac{1}{A}\left\langle\widetilde{h}_{i}^{*}\left(\mathbf{q}_{x y}^{\prime}\right) \tilde{h}_{j}\left(\mathbf{q}_{x y}^{\prime}\right)\right\rangle .
\end{aligned}
$$

The mean-square roughness $\left\langle h_{i}^{2}(0)\right\rangle$ is given by Eq. (22), and $\mathbf{q}_{x y}^{\prime}$ integration can be carried out analytically for simple resolution functions using the mean-square Fourier amplitudes in Eqs. (18) and (19). For the rectangular resolution [Eq. (34)], the resulting expressions for $\sigma_{c, i j}^{2}$ in the limit $\xi_{g}$ $\rightarrow \infty$ are given by

$$
\begin{aligned}
\sigma_{c, i i}^{2}= & \frac{k_{B} T}{2 \pi\left(\gamma_{1}+\gamma_{2}\right)}\left\{\ln \left(\frac{q_{\max }}{\Delta q_{y}}\right)\right. \\
& \left.+\frac{\gamma_{1} \gamma_{2}}{\gamma_{i}^{2}} \ln \left(\frac{\sqrt{1+\xi^{2} q_{\max }^{2}}}{\frac{1}{2} \xi \Delta q_{y}+\sqrt{1+\left(\frac{1}{2} \xi \Delta q_{y}\right)^{2}}}\right)\right\}, \\
\sigma_{c, 12}^{2}= & \frac{1}{2}\left(\sigma_{c, 11}^{2}+\sigma_{c, 22}^{2}\right)+\Delta_{c, 12}^{2},
\end{aligned}
$$

where

$$
\Delta_{c, 12}^{2}=\frac{k_{B} T}{4 \pi}\left(\frac{1}{\gamma_{1}}+\frac{1}{\gamma_{2}}\right) \ln \left[\frac{1}{2} \xi \Delta q_{y}+\sqrt{1+\left(\frac{1}{2} \xi \Delta q_{y}\right)^{2}}\right] .
$$

Using these DW factors and the intrinsic form factors $\Theta_{i}\left(q_{z}\right)$ in Eq. (3), Eq. (6) reduces to 


$$
\begin{aligned}
\frac{R\left(q_{z}\right)}{R_{F}\left(q_{z}\right)} \approx & \left|\left(\phi_{1}-1\right) e^{-(1 / 2) \sigma_{1}^{2} q_{z}^{2}}-\phi_{1} e^{-(1 / 2) \sigma_{2}^{2} q_{z}^{2}} e^{i q_{z} D}\right|^{2} \\
& -2 \phi_{1}\left(\phi_{1}-1\right)\left(e^{-\Delta_{c, 12}^{2} q_{z}^{2}}-1\right) e^{-(1 / 2)\left(\sigma_{1}^{2}+\sigma_{2}^{2}\right) q_{z}^{2}} \cos \left(q_{z} D\right),
\end{aligned}
$$

where the effective total roughness $\sigma_{i}$ at each interface is defined as $[31,34,62]$

$$
\sigma_{i}^{2} \equiv w_{i}^{2}+\sigma_{c, i i}^{2} .
$$

The first square term in Eq. (41) corresponds to the reflectivity that would be obtained if $\Delta_{c, 12}^{2}=0$, or equivalently, if the film thickness did not fluctuate ( i.e., $\xi \rightarrow q_{\max }^{-1}$ or $\left.\left\langle[\delta D(0)]^{2}\right\rangle \rightarrow 0\right)$. The second, oscillatory term arises from fluctuations in the thickness of the fluid layer.

Some features of the capillary roughness $\sigma_{c, i j}$ [Eqs. (38)-(40)] should be emphasized. First, $\sigma_{c, i j}$ depends weakly (logarithmically) on $q_{z}$ through the resolution $\Delta q_{y}$ $\approx\left(q_{z} / 2\right) \Delta \beta$, just as for $\sigma_{c}$ for the simple liquid surface (Appendix A). Second, $\sigma_{c, i j}$ depends on the film thickness $D$ through the correlation length $\xi \propto D^{2}$. Third, $\sigma_{c, i j}$ reduces to the expected single-interface forms in the extreme cases of interfacial coupling. In the limit of weak coupling or independently fluctuating interfaces, the limiting forms recover the corresponding single-interface expressions for $\sigma_{c}^{2}$ and $\left\langle h^{2}(0)\right\rangle$ [Eqs. (A6) and (A7)],

$$
\begin{gathered}
\lim _{\xi \rightarrow \xi_{g}} \sigma_{c, i i}^{2} \approx \frac{k_{B} T}{2 \pi \gamma_{i}} \ln \left(\frac{q_{\max }}{\Delta q_{y}}\right), \\
\lim _{\xi \rightarrow \xi_{g}} \sigma_{c, 12}^{2} \approx \frac{k_{B} T}{4 \pi}\left(\frac{1}{\gamma_{1}}+\frac{1}{\gamma_{2}}\right) \ln \left(q_{\max } \xi_{g}\right) \\
=\lim _{\xi \rightarrow \xi_{g}} \frac{1}{2}\left[\left\langle h_{1}^{2}(0)\right\rangle+\left\langle h_{2}^{2}(0)\right\rangle\right] .
\end{gathered}
$$

In the opposite limit of strong coupling or conformal fluctuations,

$$
\lim _{\xi \rightarrow 0} \sigma_{c, i j}^{2} \approx \frac{k_{B} T}{2 \pi\left(\gamma_{1}+\gamma_{2}\right)} \ln \left(\frac{q_{\max }}{\Delta q_{y}}\right)
$$

for all $i j$, and $\Delta_{c, 12} \rightarrow 0$ as $\xi \rightarrow 0$, consistent with $\left\langle\delta D\left(r_{x y}\right) \delta D(0)\right\rangle=0$.

The two methods described above for calculating reflectivity are compared quantitatively in Appendix B. The use of the analytic approximation based on the DW factors [Eqs. (38)-(42)] is shown to be valid so long as $q_{z}$ is sufficiently small that the exponents in Eq. (28) satisfy $\eta_{i}<1$, corresponding to $q_{z}<0.3 \AA^{-1}$ for the PFMC wetting films on C20. The calculations of $R_{i j}\left(0, q_{z}\right)$ for two nonrectangular resolution functions (Gaussian and error-function-based) are described in Appendix C.

\section{EXPERIMENTAL DETAILS}

\section{A. X-ray measurements}

$\mathrm{X}$-ray experiments were carried out at Beamline X22B of National Synchrotron Light Source, using the Harvard/BNL liquid surface spectrometer operated at an $\mathrm{x}$-ray wavelength of $\lambda=1.56 \AA$. The scattered intensities were measured using a NaI scintillation detector. Two sets of crossed Huber slits were placed between the sample and the detector, one set located at $S_{1}=233 \mathrm{~mm}$ and the other (detector slits) at $S_{2}$ $=700 \mathrm{~mm}$ from the sample center. The height and width of the slit opening at $S_{i}$ are denoted as $\left(H_{i}, W_{i}\right)$ below.

Following procedures that are now well established (see, e.g., Refs. [34,63]), the reported signal for XR is the difference between the intensity measured at the specular position $\beta=\alpha$ and $\psi=0\left[q_{x y}=0\right.$, Fig. 1(a) $]$ and the background intensity measured at $\psi$ offsets of $\pm \psi_{b}= \pm 0.3^{\circ}$. The backgroundsubtracted intensity was normalized to the incident beam to obtain the reflectivity $R\left(q_{z}\right)=I\left(q_{z}\right) / I_{0}$. The detector slits at $S_{2}$ were set to $\left(H_{2}, W_{2}\right)=(3.0 \mathrm{~mm}, 3.0 \mathrm{~mm})$, corresponding to angular detector resolutions of $\Delta \beta=0.25^{\circ}$ and $\Delta \psi=0.25^{\circ}$, or equivalently, reciprocal-space resolutions of $\Delta q_{x} \approx k \Delta \psi$ $=0.017 \AA^{-1}, \quad \Delta q_{y} \approx\left(q_{z} / 2\right) \Delta \beta=0.0021 q_{z}, \quad$ and $\quad \Delta q_{z} \approx k \Delta \beta$ $=0.017 \AA^{-1}$. The slits at $S_{1}$, set to $\left(H_{1}, W_{1}\right)$ $=(6.0 \mathrm{~mm}, 4.0 \mathrm{~mm})$, were used to reduce background and to eliminate stray scattering.

XDS was measured using a rocking scan method, in which intensities scattered in the incidence plane $(\psi=0)$ are measured as a function of the output angle $\beta$ while keeping the surface-normal component $q_{z}$ of the wave vector (or the sum $\alpha+\beta$ ) fixed. The background intensities were measured at $\psi$ offsets of $\psi_{b}= \pm 0.3^{\circ}$, and the background-subtracted intensity was normalized to the incident beam to obtain the normalized intensity $I(\alpha, \beta) / I_{0}$. The detector slits were set to $\left(H_{2}, W_{2}\right)=(0.3 \mathrm{~mm}, 3.0 \mathrm{~mm})$, corresponding to angular resolutions of $\Delta \beta=0.025^{\circ}$ and $\Delta \psi=0.25^{\circ}$. The equivalent $q$-space resolutions are given by $\Delta q_{x}=0.017 \AA^{-1}$ and $\Delta q_{z}$ $=0.0017 \AA^{-1}$, while the $q_{y}$ resolution varied with $\beta$ as $\Delta q_{y}$ $\approx k \sin (\beta) \Delta \beta=\left(0.0017 \AA^{-1}\right) \sin (\beta)$. The slits at $S_{1}$ were set to $\left(H_{1}, W_{1}\right)=(2.5 \mathrm{~mm}, 4.0 \mathrm{~mm})$. For rocking scans with fixed angular resolutions $\Delta \beta$ and $\Delta \psi, \sin (\alpha) I\left(q_{y}, q_{z}\right) / I_{0}$ is symmetric with respect to the sign of $q_{y}$ [64].

\section{B. Materials, wetting chamber, and sample preparation}

PFMC (Lancaster, 95\%) and C20 (Aldrich, 99\%) were used as received. The relevant physical parameters for the PFMC film on bulk C20 are listed in Table I [65].

The wetting chamber, sample preparation, and the method for forming wetting films are the same as in Ref. [40]. The thickness of a wetting film was controlled through the temperature difference $\Delta T=T-T_{\text {res }}>0$ between the film and a bulk reservoir of the same liquid [10,40,66,67], where the chemical potential offset $\Delta \mu$ (Sec. III A) is proportional to $\Delta T$ for small $\Delta T$. The chamber consisted of two concentric temperature-controlled cells, where the hermetically sealed inner copper cell (internal diameter $110 \mathrm{~mm}$, height $100 \mathrm{~mm}$, temperature stability $\pm 0.001 \mathrm{~K})$ contained the sample (PFMC film on bulk C20) in the middle and a reservoir of wetting liquid (PFMC) at the bottom. A circular copper disk ( 2 in. diam, $3 \mathrm{~mm}$ thick) for holding the liquid subphase (C20) was placed flat on a copper platform such that the liquid surface was approximately at $50 \mathrm{~mm}$ above the cell bottom. The platform rested on copper legs with Teflon spac- 
ers inserted in between. Two thermistors (YSI, $30 \mathrm{k} \Omega$ ) that had been calibrated against each other were used to monitor the temperature of the liquid reservoir and the sample platform. The temperature offset $\Delta T$ was controlled via an adhesive-free Kapton heater sandwiched between two plates of the sample platform assembly.

Prior to each sample preparation, the inner cell and the inside parts were wiped and/or rinsed thoroughly with chloroform and methanol, soaked in warm methanol, and dried on a heating plate. The circular copper disk for holding the sample was cleaned by wiping with methanol and soaking for $3 \mathrm{~min}$ in an acidic solution of $\mathrm{H}_{2} \mathrm{SO}_{4}(20 \%$ by volume) and $\mathrm{HNO}_{3}(10 \%)$, followed by a thorough rinse with Millipore water and drying on a heating plate. After the assembly of the inner cell, the sample platform was heated above the bulk melting point $\left(\sim 35.7^{\circ} \mathrm{C}[63]\right)$ of $\mathrm{C} 20$ and a few milligrams of $\mathrm{C} 20$ were spread uniformly over the copper disk using a spatula. The cell was then sealed without PFMC and the entire chamber was mounted on the liquid surface spectrometer. The cleanliness of the pure C20 surface was ensured by taking XR scans around the surface freezing temperature $\left(\sim 38.7^{\circ} \mathrm{C}\right)$ and checking that the surface freezing property of $\mathrm{C} 20$ [63] was reproducible.

For the formation of a wetting film, a reservoir of PFMC was introduced by opening a small screw hole (diameter $1.5 \mathrm{~mm}$ ) on the cell lid, injecting $3 \mathrm{~mL}$ of PFMC to the cell bottom via a Hamilton syringe, and immediately resealing the hole. After setting the temperatures for given film temperature and offset $\Delta T$, the sample was left undisturbed until the temperatures stabilized, typically for $1-2 \mathrm{~h}$, prior to starting X-ray measurements. At each temperature setting, XR measurements were repeated multiple times over a few hours to ensure that the wetting film was stable. With this setup, it was possible to obtain stable PFMC films up to $D$ $\sim 160 \AA[40]$.

\section{EXPERIMENTAL RESULTS, ANALYSIS, AND DISCUSSIONS}

\section{A. Specular reflectivity}

Representative XR data from PFMC wetting films on C20 at 37 and $42{ }^{\circ} \mathrm{C}$ are shown as $R\left(q_{z}\right) / R_{F}\left(q_{z}\right)$ in Figs. 4(a) and 4(b), respectively. The shown data sets represent films of different thicknesses $(D=37$ to $142 \AA)$, obtained at different temperature offsets $\Delta T$ between the $\mathrm{C} 20$ subphase and the PFMC reservoir. The period (in $q_{z}$ ) of the interference (Kiessig) fringe is a direct measure of the inverse thickness $D^{-1}$, and the topmost (bottommost) data set in each figure corresponds to the thinnest (thickest) film, respectively. The $1 / 3$ power-law behavior $D \sim(\Delta T)^{-1 / 3}$ of PFMC wetting films on $\mathrm{C} 20$, which signifies complete wetting, has already been discussed in Ref. [40]. The primary goal of the present XR study is to extract the interfacial structures and their dependence on the interfacial separation $D$.

Since the kinematic Born approximation (BA) is not accurate for $q_{z} / q_{c} \lesssim 5[31]\left(q_{c}=0.0195 \AA^{-1}\right.$ for bulk C20), the analysis of the XR data has been carried out using different formalisms for $q_{z} \geqslant 0.1 \AA^{-1}\left(\sim 5 q_{c}\right)$ and for $q_{z}<0.1 \AA^{-1}$. For

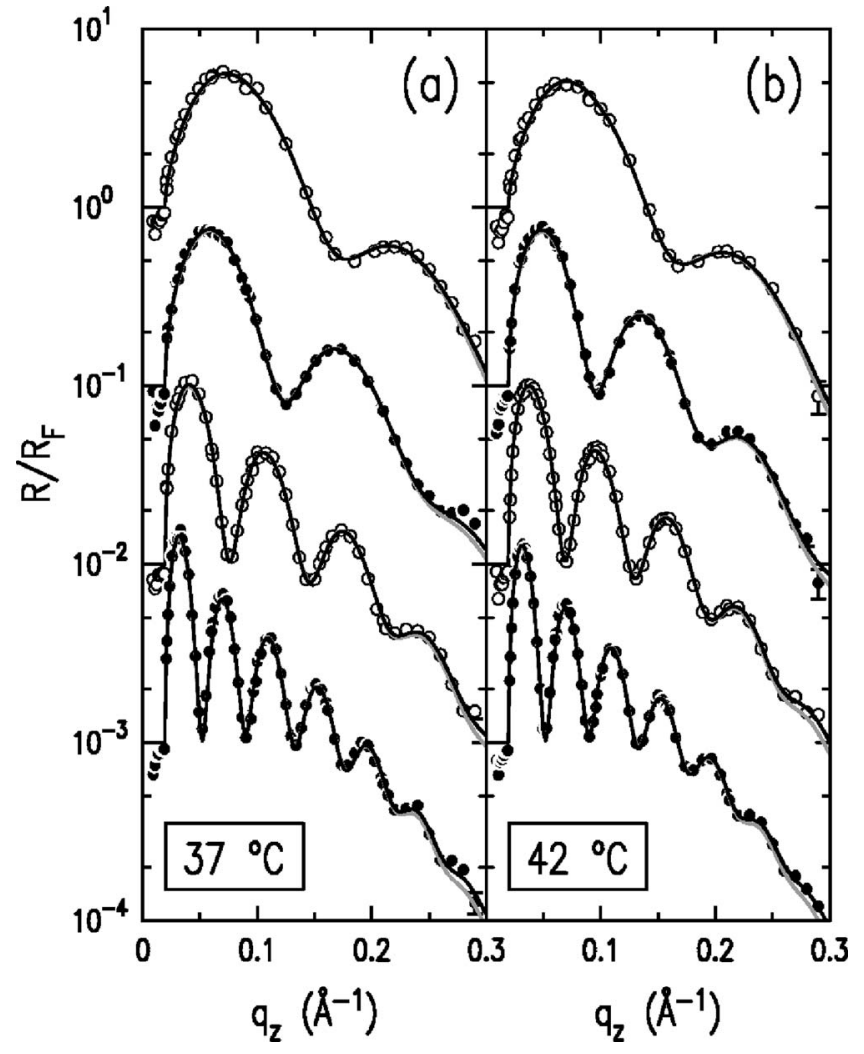

FIG. 4. Measured Fresnel-normalized specular reflectivity $R / R_{F}$ vs $q_{z}$ (symbols), obtained from PFMC wetting films on bulk C20: (a) at $37.2^{\circ} \mathrm{C}$ and thickness $D=37,51,87$, and $142 \AA$ (top to bottom); and (b) at $42.2{ }^{\circ} \mathrm{C}$ and $D=38,66,97$, and $142 \AA$ (top to bottom). Each data set is accompanied by the best-fit $R / R_{F}$ curves based on fit 1 (the gray line) and fit 2 (the black line) of the XR analysis; the two fits are almost identical except for the slight differences at high $q_{z}$.

$q_{z} \geqslant 0.1 \AA^{-1}$, the fitting curve was calculated by using the BA expression based on the DW factors [Eq. (41)]. As shown in Appendix B, this BA form should be accurate for the PFMC-C20 system up to $q_{z} \sim 0.3 \AA^{-1}$, i.e., the highest $q_{z}$ probed in the present measurement. In the fitting, the four parameters $D, \phi_{1}, \sigma_{1}$, and $\sigma_{2}$ were varied. The factor $\Delta_{c, 12}$ $\left(\rightarrow 0\right.$ as $\left.q_{z} \rightarrow 0\right)$ in the correlation correction term in Eq. (41) was calculated from Eq. (40), using the known resolution $\Delta q_{y}=\left(q_{z} / 2\right) \Delta \beta$, the appropriate correlation length $\xi$ for given $D$ [Eq. (20)], and the parameters in Table I.

For $q_{z}<0.1 \AA^{-1}$, the reflectivity was estimated by a combination of the Parratt formalism $[1,68,69]$ and the BA. For the lowest values $q_{z}<0.06 \AA^{-1}$, the fitting curve was calculated by replacing the first (square) term in Eq. (41) with the $R / R_{F}$ based on the matrix method of the Parratt formalism [69]. For this calculation, a single-layer electron density profile ("box model;" see, e.g., Refs. $[48,50,66])$ was first constructed from the same four parameters $\left(D, \phi_{1}, \sigma_{1}\right.$, and $\left.\sigma_{2}\right)$ as used in the BA; the profile was then divided into a stack of constant-density, 0.5 - $\AA$-thick slabs, and the exact reflectivity was calculated for this set of slabs. Unfortunately, there is a systematic error in the BA analysis that is due to neglecting refraction effects and is responsible for a small difference between the Parratt and BA forms [70]. This difference is not 
TABLE II. List of best-fit parameters, based on fit 1 , for fitting to the XR data in Fig. 4.

\begin{tabular}{|c|c|c|c|}
\hline $\begin{array}{l}D \\
(\AA)\end{array}$ & $\phi_{1}=\rho_{1} / \rho_{\infty}$ & $\begin{array}{c}\sigma_{1}(L / L) \\
(\AA)\end{array}$ & $\begin{array}{c}\sigma_{2}(L / V) \\
(\AA)\end{array}$ \\
\hline \multicolumn{4}{|c|}{ (a) $T=37.2{ }^{\circ} \mathrm{C}$} \\
\hline $36.7 \pm 0.4$ & $1.84 \pm 0.03$ & $8.1 \pm 0.5$ & $6.22 \pm 0.12$ \\
\hline $50.5 \pm 0.3$ & $1.90 \pm 0.03$ & $8.9 \pm 0.3$ & $6.38 \pm 0.10$ \\
\hline $86.4 \pm 0.6$ & $1.89 \pm 0.05$ & $9.2 \pm 0.4$ & $6.29 \pm 0.18$ \\
\hline $141.9 \pm 0.8$ & $1.90 \pm 0.05$ & $9.8 \pm 0.7$ & $6.40 \pm 0.20$ \\
\hline \multicolumn{4}{|c|}{ (b) $T=42.2{ }^{\circ} \mathrm{C}$} \\
\hline $38.0 \pm 0.5$ & $1.77 \pm 0.03$ & $8.3 \pm 0.5$ & $6.47 \pm 0.15$ \\
\hline $65.8 \pm 0.5$ & $1.81 \pm 0.03$ & $9.1 \pm 0.4$ & $6.41 \pm 0.13$ \\
\hline $96.9 \pm 0.5$ & $1.85 \pm 0.03$ & $9.4 \pm 0.5$ & $6.45 \pm 0.12$ \\
\hline $141.9 \pm 0.6$ & $1.83 \pm 0.03$ & $10.3 \pm 0.5$ & $6.49 \pm 0.13$ \\
\hline
\end{tabular}

physically important, and in order to provide a smooth transition in the region $0.06<q_{z}<0.1 \AA^{-1}$, the square term in Eq. (41) was replaced by a weighted sum of the Parratt and BA forms, where the relative weight of the BA contribution was increased linearly from 0 to 1 over this $q_{z}$ range.

The theoretical $R / R_{F}$ curve based on these high- $q_{z}$, low$q_{z}$, and intermediate forms was fitted simultaneously to the observed data in the range $q_{z}>0.03 \AA^{-1}$. For all $q_{z}>0$, the calculated $R / R_{F}$ contained the BA-based correlation correction term [Eq. (41)], which vanishes at $q_{z}=0$. The actual fitting was carried out using two different types of parametrizations. The two methods, denoted hereafter as fit 1 and fit 2, differ in whether the total roughnesses $\sigma_{i}$ [Eq. (42)] or the intrinsic widths $w_{i}$ are treated as fitting parameters. Both approaches produce best fits of similarly good quality.

\section{Fit 1: Effective total roughness $\sigma_{i}$}

In fit 1 , the weak $q_{z}$ dependence of $\sigma_{i}$ (through $\sigma_{c, i i}$ ) is completely neglected, and $\sigma_{i}$ are simply treated as fitting parameters. Thus, the four directly varied parameters are $D$, $\phi_{1}, \sigma_{1}$, and $\sigma_{2}$. The best-fit $R / R_{F}$ curves obtained by fit 1 are indicated by the gray lines in Fig. 4, and the best-fit parameters for the shown curves are listed in Table II. The presence of a nearly immiscible PFMC wetting film on C20 is supported by the fact that the extracted values for the relative electron density $\phi_{1}$ of the layer agree, within $7 \%$ or less, with the expected nominal value of $\phi_{1}=\rho_{\mathrm{PFMC}} / \rho_{\mathrm{C} 20} \sim 1.9$. The best-fit effective total roughness $\sigma_{i}$ are plotted as a function of the film thickness $D$ in Fig. 5, where the upper data set corresponds to the liquid-liquid interface $\left(\sigma_{1}\right)$ and the lower set to the liquid-vapor interface $\left(\sigma_{2}\right)$. The observed relation $\sigma_{1}>\sigma_{2}$ is consistent with the lower surface tension $\left(\gamma_{1}<\gamma_{2}\right)$ of the liquid-liquid interface as compared to that of the liquid-vapor interface.

For comparison, Fig. 5 also shows three different sets of theoretical curves for the capillary wave roughness $\sigma_{c, i i}$. The black solid curves correspond to the expected case of coupled interfacial fluctuations mediated by vdW interactions [Eq. (38)]. The two horizontal solid lines in gray correspond to the limit of weak interfacial coupling, i.e., two

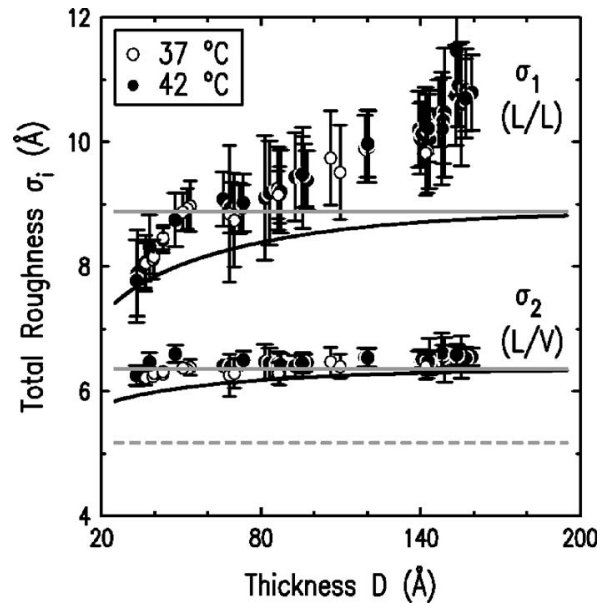

FIG. 5. Effective total roughness $\sigma_{i}=\sqrt{w_{i}^{2}+\sigma_{c, i i}^{2}}$ as a function of PFMC film thickness $D$ at $37.2{ }^{\circ} \mathrm{C}(\bigcirc)$ and $42.2^{\circ} \mathrm{C}(\bullet)$, representing the best-fit values extracted from fit 1 of the XR analysis. The upper data set corresponds to $\sigma_{1}$ for the C20-PFMC (liquid-liquid) interface and the lower set to $\sigma_{2}$ for the PFMC-vapor (liquid-vapor) interface. The lines describe the theoretical effective capillary roughness $\sigma_{c, i i}$ for three different scenarios of interfacial coupling, calculated using the physical parameters in Table I and a fixed resolution of $\Delta q_{y}=\left(q_{z} / 2\right) \Delta \beta=3.2 \times 10^{-4} \AA^{-1}$ at a midpoint $q_{z}$ $=0.15 \AA^{-1}$ of the XR measurement. The black solid lines that vary with $D$ are based on Eq. (38) and correspond to the expected coupling of interfacial fluctuations that is mediated by (nonretarded) $\mathrm{vdW}$ interactions. The two horizontal solid lines in gray describe the limit of weak coupling or independent fluctuations [Eq. (43)]. The bottommost gray dashed line represents the limit of strong coupling or perfectly conformal fluctuations [Eq. (45)].

independently fluctuating interfaces [Eq. (43)]. The gray dashed line on the bottom describes the limit of strong coupling, in which two interfaces fluctuate together and obey perfect conformality so that $\sigma_{c, 11}$ (conf.) $=\sigma_{c, 22}$ (conf.) [Eq. (45)]. All these curves were calculated by using the parameters in Table I and assuming the resolution of $\Delta q_{y}=3.2$ $\times 10^{-4} \AA^{-1}$ at $q_{z}=0.15 \AA^{-1}$. The upper wave-vector cutoff, on which $\sigma_{c, i i}^{2}$ depends logarithmically (Sec. III D), was fixed at $q_{\max }=\pi / a=0.5 \AA^{-1}$, on the basis of $a \sim 6 \AA$ for the diameter of the PFMC molecule [66]. This value is also close to the published value of $q_{\max }=0.44 \pm 0.06 \AA^{-1}$ for the free surface of bulk C20 [34]. In Fig. 5, the capillary roughness $\sigma_{c, i i}(\mathrm{vdW})$ for the vdW-coupled fluctuations displays the expected $D$-dependent behavior $[12,15,16,37,66]$. At small $D$, $\sigma_{c, i i}(\mathrm{vdW})$ is partially quenched below the value $\sigma_{c, i i}$ (indep.) for the independent fluctuations due to the close proximity of the two interfaces; however, as $D$ increases, $\sigma_{c, i i}(\mathrm{vdW})$ increases and saturates to $\sigma_{c, i i}$ (indep.) at large $D$.

On the basis of Fig. 5, the following inferences can be made. (i) The observed total roughness $\sigma_{i}$ at each interface is dominated by the capillary roughness $\sigma_{c, i i}$. (ii) While the possibility of perfectly conformal fluctuations is clearly ruled out, the measured roughnesses $\sigma_{i}$ are of the same order of magnitude as both $\sigma_{c, i i}(\mathrm{vdW})$ for the vdW-coupled fluctuations and $\sigma_{c, i i}$ (indep.) for the independent fluctuations. In view of this, the XR result here is not sufficient justification for discriminating unambiguously between the coupled and 
TABLE III. List of best-fit parameters, based on fit 2, for fitting to the XR data in Fig. 4.

\begin{tabular}{|c|c|c|c|}
\hline $\begin{array}{l}D \\
(\AA)\end{array}$ & $\phi_{1}=\rho_{1} / \rho_{\infty}$ & $\begin{array}{c}w_{1}(L / L) \\
(\AA)\end{array}$ & $\begin{array}{c}w_{2}(L / V) \\
(\AA)\end{array}$ \\
\hline \multicolumn{4}{|c|}{ (a) $T=37.2{ }^{\circ} \mathrm{C}$} \\
\hline $36.6 \pm 0.4$ & $1.86 \pm 0.03$ & $3.2 \pm 1.1$ & $2.3 \pm 0.3$ \\
\hline $50.6 \pm 0.3$ & $1.93 \pm 0.03$ & $4.3 \pm 0.6$ & $2.4 \pm 0.2$ \\
\hline $86.5 \pm 0.6$ & $1.91 \pm 0.05$ & $4.1 \pm 1.2$ & $1.8 \pm 0.6$ \\
\hline $142.0 \pm 0.8$ & $1.92 \pm 0.05$ & $5.0 \pm 1.3$ & $1.8 \pm 0.7$ \\
\hline \multicolumn{4}{|c|}{ (b) $T=42.2^{\circ} \mathrm{C}$} \\
\hline $37.9 \pm 0.5$ & $1.79 \pm 0.03$ & $3.1 \pm 1.3$ & $2.7 \pm 0.4$ \\
\hline $65.7 \pm 0.4$ & $1.83 \pm 0.03$ & $3.9 \pm 0.9$ & $2.2 \pm 0.3$ \\
\hline $96.9 \pm 0.5$ & $1.86 \pm 0.02$ & $4.0 \pm 0.9$ & $2.0 \pm 0.4$ \\
\hline $142.0 \pm 0.7$ & $1.85 \pm 0.03$ & $5.5 \pm 1.1$ & $1.9 \pm 0.5$ \\
\hline
\end{tabular}

independent fluctuations. We will return to this point below when we discuss XDS results. On the other hand, the fact that the measured $\sigma_{i}$ remain close to or above $\sigma_{c, i i}(\mathrm{vdW})$ does point toward the plausibility of the vdW-coupled capillary fluctuations and small but non-negligible intrinsic widths $w_{i}$. (iii) The observed $\sigma_{1}$ is consistently higher than $\sigma_{c, 11}(\mathrm{vdW})$, although only by small amounts for the smallest $D$ probed. This suggests that the intrinsic width $w_{1}$ at the liquid-liquid (C20-PFMC) interface is nonzero. This point, as well as the following, is better addressed by the second fitting method to be discussed below. (iv) The fact that the observed $\sigma_{2}$ is fairly close to $\sigma_{c, 22}(\mathrm{vdW}) \approx \sigma_{c, 22}$ (indep.) at all $D$, implies that the intrinsic width $w_{2}$ of the liquid-vapor (PFMC-vapor) interface is smaller than $w_{1}$ and remains so for all $D$.

\section{Fit 2: Intrinsic widths $w_{i}$}

In fit 2, the capillary roughnesses $\sigma_{c, i i}$ are assumed to be given by $\sigma_{c, i i}(\mathrm{vdW})$ for the coupled interfacial fluctuations and are calculated exactly using Eq. (38). Therefore, their dependence on $q_{z}$ through the resolution $\Delta q_{y}$ is completely accounted for, and the only unknown in the calculation of $\sigma_{c, i i}(\mathrm{vdW})$ is the film thickness $D$, which is a fitting parameter. Thus, for fit 2, the total roughnesses $\sigma_{i}$ are varied only indirectly, and the four directly varied parameters are $D, \phi_{1}$, and the intrinsic widths $w_{1}$ and $w_{2}$. The best-fit $R / R_{F}$ curves are shown as black lines in Fig. 4, and the corresponding best-fit parameters are listed in Table III. The quality of the fits is similar to that for fit 1 , except that fit 2 tends to reproduce the data slightly better than fit 1 at high $q_{z}$. The two fitting methods yield the values for $D$ and $\phi_{1}$ that agree within their uncertainties. The best-fit intrinsic widths $w_{i}$ are plotted as a function of $D$ in Fig. 6.

While the uncertainties are relatively large, the behavior of the extracted intrinsic widths $w_{i}$ is consistent with the inferences made earlier in Sec. V A 1. Figure 6(a) indicates that $w_{1}$ at the liquid-liquid (C20-PFMC) interface is nonzero, with the best-fit values in the range 2-6 $\AA$. The previous XR study of water-alkane interfaces by Mitrinovic et al. [38] has provided evidence that the intrinsic width of an immiscible,

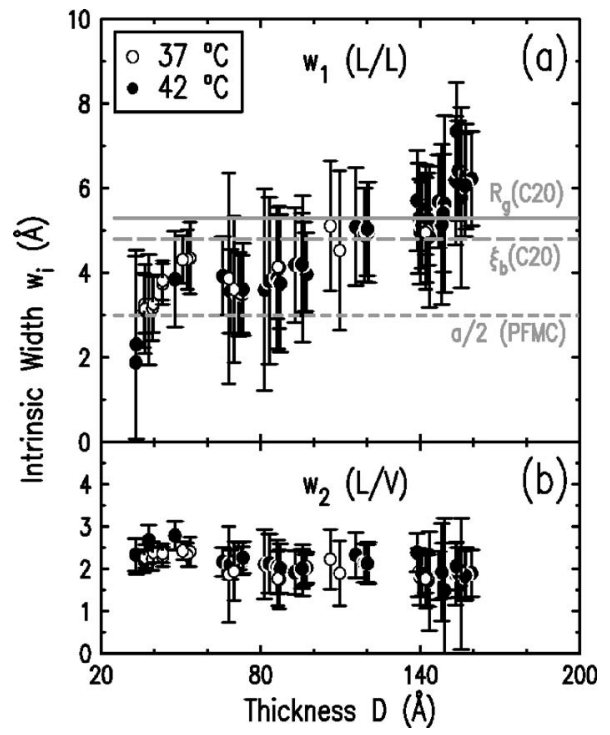

FIG. 6. Intrinsic or local width (a) $w_{1}$ of the C20-PFMC (liquidliquid) interface and (b) $w_{2}$ of the PFMC-vapor (liquid-vapor) interface, as a function of PFMC film thickness $D$ at $37.2^{\circ} \mathrm{C}(\bigcirc)$ and $42.2^{\circ} \mathrm{C}(\bigcirc)$. The symbols represent the best-fit values extracted from fit 2 of the XR analysis. In (a), the gray horizontal lines correspond to the radius of gyration $R_{g} \approx 5.3 \AA$ for $\mathrm{C} 20$ (solid line) [34], the bulk correlation length $\xi_{b} \approx 4.8 \AA$ for long-chain alkanes (long dashed line) [38], and the molecular radius $a / 2 \sim 3 \AA$ for PFMC (short dashed line) [66].

bulk liquid-liquid interface (i.e., $D \rightarrow \infty$ ) is determined by either the molecular size or the bulk correlation length associated with the larger of the two molecular components. In particular, the intrinsic width that they extracted for the water-alkane interface was found to agree with the radius of gyration $R_{g}$ of the alkane molecule for short-chain alkanes (Cn with $n \lesssim 16$ ) and with the bulk correlation length $\xi_{b}$ of liquid alkane for long-chain alkanes $(n \geq 16)$. For similar comparison, Fig. 6(a) shows three horizontal lines representing the gyration radius $R_{g} \approx 5.3 \AA$ for C20 (solid line) [34], an estimated bulk correlation length $\xi_{b} \approx 4.8 \AA$ for molten long-chain alkanes (long dashed line) [38], and the molecular radius $a / 2 \approx 3 \AA$ for PFMC (short dashed line) [66]. Due to the large uncertainties, it is not possible to discern whether the measured width $w_{1}$ is in better agreement with $R_{g}$ or $\xi_{b}$ for C20. However, the fact that $w_{1}$ observed for large $D$ $(\geq 50 \AA)$ agrees, within error, with these two lengths associated with $\mathrm{C} 20$ is consistent with the expected behavior of the bulk liquid-liquid interface. Another interesting observation is that $w_{1}$ for $D \leq 50 \AA$ is consistently smaller than $R_{g}$ or $\xi_{b}$ for $\mathrm{C} 20$, possibly indicating a reduction in the intrinsic liquid-liquid interfacial width for very thin films. Although the uncertainties associated with this observation are relatively large, it is interesting to note that a similar thicknessdependent or confinement-induced reduction of the intrinsic width at a buried liquid-liquid interface has been observed in thin binary wetting films on a solid substrate [66].

By contrast, Fig. 6(b) shows that the intrinsic width $w_{2}$ of the liquid-vapor interface is always small $\left(w_{2}=2 \pm 1 \AA\right)$ and remains nearly constant as the interfacial separation $D$ is varied. The narrow width of the PFMC-vapor interface is 
consistent with the general observation that the intrinsic width of a liquid-vapor interface is usually no more than the size of atoms, as demonstrated previously for the surface of bulk water [32,33,46], ethanol [29], and normal alkanes [34], as well as for the free surface of organic and polymer liquid films on silicon substrates $[12,16,66]$.

\section{B. Off-specular diffuse scattering}

Due to its $q_{y}$ dependence, XDS is better suited than XR for probing the effect of interfacial coupling, which should manifest itself through the lateral correlation length $\xi$. In order to effectively discriminate between the vdW-coupled and independent fluctuations, the dynamic range (in $q_{y}$ ) of the XDS measurement must be sufficiently wide and the correlation length $\xi \propto D^{2}$ [Eq. (20)] must be long enough to meet the condition $\Delta q_{y} \ll \xi^{-1} \ll \max \left\{\left|q_{y}\right|\right\}$. This condition is satisfied in the present experiments for the thickest stable films of $D \sim 150 \AA(\xi \sim 4000 \AA)$, provided that the fixed $q_{z}$ for a rocking scan is roughly in the range $0.1-0.15 \AA^{-1}$. The restriction in $q_{z}$ arises for two reasons. First, the range of rocking scans is limited to a maximum value of $\max \left\{\left|q_{y}\right|\right\}$ $\sim q_{z}^{2} / 2 k$, and if $q_{z}<0.1 \AA^{-1}$, the dynamic range of the scans is too small to gain a true measure of the algebraic cusp. Second, the upper limit on $q_{z}$ arises because the XDS intensities are significantly reduced for higher $q_{z}$. Within this range, the exact value of the fixed $q_{z}$ should be chosen such that the difference in the expected $I / I_{0}$ between the two types of fluctuations is maximized. This is best done by comparing rocking scans that are measured at $q_{z}$ for minima and maxima of the Kiessig fringes in $R\left(q_{z}\right) / R_{F}\left(q_{z}\right)$ [i.e., $\left.\cos \left(q_{z} D\right)= \pm 1\right]$ [22]. As discussed in Sec. III C, for the independent fluctuations, the $R_{12}\left(q_{y}, q_{z}\right)$ term makes no contribution to $I / I_{0}$ at $\left|q_{y}\right| \gg \Delta q_{y}$ [Eq. (32)]. Therefore, observed differences between these scans would provide a direct indication of nonindependent fluctuations.

The results of rocking scans from films at $D=140,148$, and $155 \AA$ are shown as open symbols in the lower panels of Figs. 7(a)-7(c), respectively, in terms of the normalized intensity $\sin (\alpha) I\left(q_{y}, q_{z}\right) / I_{0}$. The $q_{z}$ position of each scan is close to either a maximum or a minimum in $R / R_{F}$, as indicated in the upper panels that show the XR data taken immediately prior to the XDS scans. The XDS data for $q_{y}<0$ and $q_{y}>0$ are plotted on top of each other and show good overlap between the two sides as expected for fixed- $q_{z}$ scans [64]. Each rocking scan is characterized roughly by three regions: the specular part at small $\left|q_{y}\right|<\Delta q_{y}$, the off-specular part at larger $\left|q_{y}\right|>\Delta q_{y}$, and a surface enhancement ("Yoneda") peak near $\max \left\{\left|q_{y}\right|\right\}$, which occurs at $\alpha$ or $\beta$ $\sim \alpha_{c}$ [through $T_{F}(\theta)$ in Eq. (2)] $[28,29]$. The off-specular scattering at reflectivity maxima $[\triangle$ in Figs. $7($ a) and $7(\mathrm{c})]$ falls off monotonically with increasing $\left|q_{y}\right|$, with a constant slope in the log-log plot. By contrast, the falloff of the offspecular scattering at reflectivity minima $[\bigcirc$ in Figs. 7(a) and 7(b)] appears to change its slope in the log-log plot near $\left|q_{y}\right|=\xi^{-1}$. A similar kink in the XDS rocking scan has been observed for thin organic and polymer liquid films on silicon substrates $[12,14-16]$. This is a direct demonstration that the correlation length $\xi$ affects the scattering behavior and pro- vides qualitative evidence for the expected presence of $\mathrm{vdW}$ coupled capillary fluctuations at the two interfaces.

For quantitative comparison, the predicted normalized intensities $\sin (\alpha) I / I_{0}$ are shown as thick, gray solid lines in Fig. 7. The factors $R_{i j}\left(q_{y}, q_{z}\right)$ for the vdW-coupled fluctuations were calculated by performing the numerical integration in Eq. (30) for the Gaussian resolution function, using the approximate forms of $\exp \left[-\frac{1}{2} g_{i j}\left(r_{x y}\right) q_{z}^{2}\right]$ in Eqs. (26) and (27), the physical parameters in Table I, and the experimental resolution $\Delta q_{y}=k \sin (\beta) \Delta \beta$ that corresponds to the nominal XDS slit setting (Sec. IV A). The remaining parameters $(D$, $\phi_{1}, w_{1}, w_{2}$ ) were obtained from the fit 2 analysis of the associated $R / R_{F}$ data. As a result, the calculation of $I / I_{0}$ involves no adjustable parameters. Figures 7(b) and 7(c) also show the two limiting cases of theoretical off-specular scattering for $\left|q_{y}\right| \gg \Delta q_{y}$, where the black solid and dashed lines correspond to the independent and perfectly conformal fluctuations, respectively. These $I / I_{0}$ curves are based on the asymptotic forms of $R_{i j}\left(q_{y}, q_{z}\right)$ in Eqs. (31)-(33) and, again, calculated with no adjustable parameters.

It is clear from Fig. 7 that the measured XDS data are consistent with the vdW-coupled capillary fluctuations of the two interfaces. The predicted $I / I_{0}$ curves reproduce not only the uniform falloff in the data for $q_{z}$ at the $R / R_{F}$ maxima but also the observed crossover behavior around $\left|q_{y}\right| \sim \xi^{-1}$ for $q_{z}$ at the $R / R_{F}$ minima. By contrast, regardless of the $q_{z}$ position, the independent and perfectly conformal fluctuations would produce only a uniform falloff in $I / I_{0}$. Moreover, for $q_{z}$ at the $R / R_{F}$ minima, the independent fluctuations would overestimate $I / I_{0}$ for $\left|q_{y}\right|<\xi^{-1}$, while the conformal fluctuations would underestimate $I / I_{0}$ especially at large $\left|q_{y}\right|$.

Finally, it should be noted that the XDS results presented here support the reliability of the fit 2 analysis of the XR data, from which the intrinsic interfacial widths $w_{i}$ have been extracted by assuming the vdW-coupled capillary fluctuations.

\section{SUMMARY}

The thermal capillary fluctuations and the intrinsic widths of two interacting fluid-fluid interfaces have been studied by a combination of x-ray specular reflectivity and off-specular diffuse scattering measurements. The primary objective of this study has been to probe for the expected vdW-dominated coupling of capillary fluctuations at the liquid-liquid and liquid-vapor interfaces of PFMC wetting films on the surface of bulk C20. The analysis of the XR and XDS data indicates that the observed magnitudes and wave-vector dependence of specular and off-specular intensities are consistent with the presence of the vdW-coupled capillary fluctuations, provided that each interface possesses a small but finite intrinsic width $w_{i} \neq 0$. The presence of the coupled capillary fluctuations is evidenced most convincingly by the fact that the XDS rocking scans taken at $q_{z}$ near $R / R_{F}$ minima display a crossover behavior near $\left|q_{y}\right| \sim \xi^{-1}$, i.e., the expected inverse correlation length for the vdW-mediated film thickness fluctuations. Moreover, we have demonstrated that both this crossover behavior and its absence at $R / R_{F}$ maxima are well reproduced by the theoretically calculated intensity that takes 

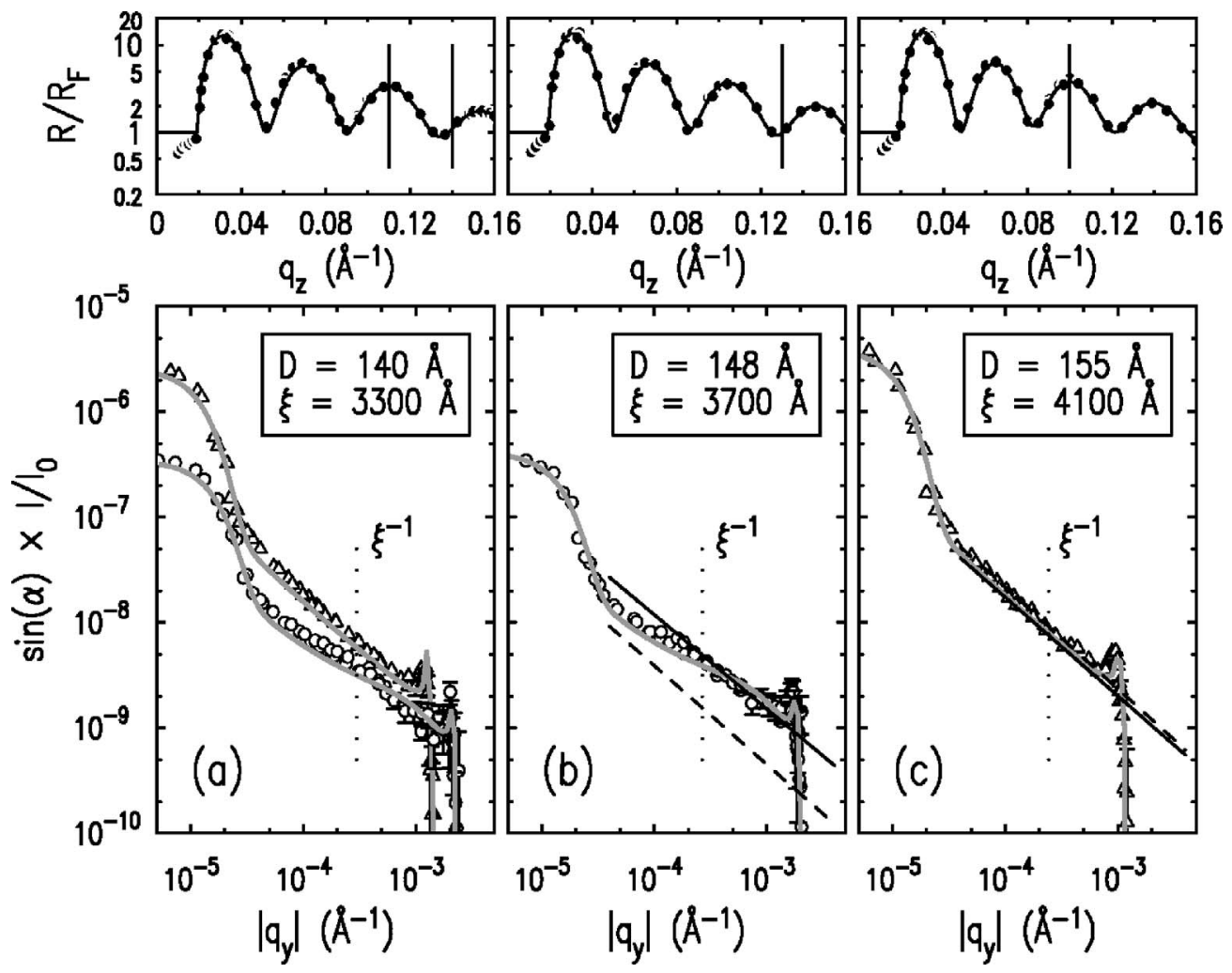

FIG. 7. Demonstration of the vdW-dominated coupling of capillary fluctuations at the liquid-liquid and liquid-vapor interfaces. The lower panels show measured off-specular diffuse rocking scans in the incidence plane $\left(q_{x}=0, \psi=0\right)$, from PFMC wetting films on bulk C20 at $42.2^{\circ} \mathrm{C}$ : (a) $D=140 \AA(\xi=3300 \AA)$ at $q_{z}=0.11 \AA^{-1}(\triangle)$ and $0.14 \AA^{-1}(\bigcirc)$; (b) $D=148 \AA(\xi=3700 \AA)$ at $q_{z}=0.13 \AA^{-1}(\bigcirc)$; and (c) $D$ $=155 \AA(\xi=4100 \AA)$ at $q_{z}=0.10 \AA^{-1}(\triangle)$. The data for both $q_{y}>0$ and $q_{y}<0$ are plotted on top of each other in terms of the normalized intensity $\sin (\alpha) I / I_{0}$ vs $\left|q_{y}\right|$. The vertical dotted line indicates the position of the expected inverse correlation length $\xi^{-1}$. For each $D$, the upper panel shows the associated $R / R_{F}$ data $(\bullet)$ obtained immediately before the diffuse scan, together with the corresponding best-fit curve based on fit 2 (solid curve). The fixed $q_{z}$ position of each rocking scan is close to either a maximum or a minimum in $R / R_{F}$, as indicated in the upper panels. In the lower panels, the thick solid lines in gray represent the theoretical $\sin (\alpha) I / I_{0}$ for the expected vdW-mediated coupling of interfacial fluctuations. The calculation, which involves no adjustable parameters, is based on the expressions for $I / I_{0}$ and $\Theta_{i}\left(q_{z}\right)$ in Eqs. (2) and (3); the factors $R_{i j}\left(q_{y}, q_{z}\right)$ in Eq. (30) for the Gaussian resolution function with the experimental $\Delta q_{y}=k \sin (\beta) \Delta \beta$; the approximate correlation functions in Eqs. (26) and (27); the physical parameters in Table I; and the best-fit parameters $\left(D, \phi_{1}, w_{1}\right.$, and $\left.w_{2}\right)$ from the fit 2 analysis of the XR data. In (b) and (c), the black solid lines represent the limit of weak coupling or independent interfacial fluctuations [via the asymptotic forms of $R_{i j}\left(q_{y}, q_{z}\right)$ in Eqs. (31) and (32)]; the black dashed lines are for the limit of strong coupling or perfectly conformal fluctuations [via the asymptotic form of $R_{i j}\left(q_{y}, q_{z}\right)$ in Eq. (33)].

the effects of $\xi$ into account. The intrinsic widths of the two interfaces have been extracted from the XR data analysis by theoretically accounting for the effects of the vdW-coupled capillary fluctuations on $R / R_{F}$. The intrinsic width of the liquid-liquid (C20-PFMC) interface for $D \gtrsim 50 \AA$ is found to be comparable to the value expected for the bulk liquidliquid interface $(D \rightarrow \infty)$, determined by either the gyration radius of the $\mathrm{C} 20$ molecule $(5.3 \AA$ [34]) or the bulk correlation length in molten long-chain alkanes (4.8 $\AA$ [38]); for $D \lesssim 50 \AA$, the width appears to be reduced below these expected bulk values, possibly indicating the effect of confinement. By contrast, the liquid-vapor (PFMC-vapor) interface has a sharper intrinsic width of $w_{2}=2 \pm 1 \AA$ that is essentially independent of $D$.

Another major objective of the present study has been to develop a relatively simple and practical approach for estimating the XR and XDS intensities that fully account for both the subtlety of liquid surface scattering and the complexity associated with the partially correlated capillary roughness of the two fluid-fluid interfaces. For this purpose, we presented two different methods for approximating the theoretical intensities and illustrated their practical utility and accuracy by applying them to the data analysis. The first method, which is more useful for XDS than XR, is to make use of approximate behavior of the height-height and thickness-thickness correlation functions. Despite their simplicity, the approximate correlation functions that we used [Eqs. (22)-(27), Fig. 2] capture the basic effects of the coupled capillary fluctuations of the two interfaces. The combination of these correlation functions and the realistic resolution functions that closely represent actual experiments can be used to calculate the normalized intensities $I / I_{0}$ for XDS (Sec. III C) and XR (Sec. III D). The second approximation method, discussed in Sec. III D, results in a simple analytic form of $R / R_{F}$ [Eq. (41)] that is based on DebyeWaller factors and effective capillary roughnesses [Eqs. (38)-(40)]. This approximate form of $R / R_{F}$ properly accounts for the effects of both the resolution and the coupled 
capillary fluctuations of the two interfaces. Its use in the XR analysis should be valid for $q_{z} / q_{c} \gtrsim 5$ so long as both of the two exponents satisfy $\eta_{i}=\left(k_{B} T / 2 \pi \gamma_{i}\right) q_{z}^{2}<1$ (Appendix B, Fig. 8). The various expressions that we presented for the correlation functions, the scattering intensities and the effective capillary roughness all reduce to the proper forms in the limit of weak and strong interfacial coupling, corresponding to the limits of independent and perfectly conformal fluctuations.

\section{ACKNOWLEDGMENTS}

We gratefully acknowledge Christopher Rulison of Kruss USA for the surface tension measurement of the bulk liquidliquid interface. The Harvard contribution to this work was supported by Grant No. NSF-DMR-01-24936. The work at Brookhaven National Laboratory was supported by the U.S. Department of Energy under Contract No. DE-AC0298CH10886. Use of National Synchrotron Light Source, Brookhaven National Laboratory, was supported by the U.S. Department of Energy, under Contract No. DE-AC0298CH10886.

\section{APPENDIX A: LIQUID SURFACE SCATTERING IN THE SINGLE-INTERFACE LIMIT}

For solid surfaces, a common approach for estimating scattered intensities is to decompose Eq. (2) into the true specular term $\left[d \sigma / d \Omega \sim \delta^{(2)}\left(\mathbf{q}_{x y}\right)\right.$ or $\left.I / I_{0} \sim \Xi\left(\mathbf{q}_{x y}\right)\right]$ and the diffuse contribution through Eq. (4) $[24,28,41-44]$,

$$
\begin{aligned}
R_{i j}\left(\mathbf{q}_{x y}, q_{z}\right) \approx & e^{-(1 / 2)\left[\left\langle h_{i}^{2}(0)\right\rangle+\left\langle h_{j}^{2}(0)\right\rangle\right] q_{z}^{2}} \\
& \times\left\{\Xi\left(\mathbf{q}_{x y}\right)+\frac{1}{(2 \pi)^{2}} \int d^{2} \mathbf{q}_{x y}^{\prime} \Xi\left(\mathbf{q}_{x y}-\mathbf{q}_{x y}^{\prime}\right)\right. \\
& \times \int_{A} d^{2} \mathbf{r}_{x y} e^{\left.i \mathbf{q}_{x y}^{\prime} \cdot \mathbf{r}_{x y}\left[e^{\left\langle h_{i}\left(\mathbf{r}_{x y}\right) h_{j}(0)\right\rangle q_{z}^{2}}-1\right]\right\} .}
\end{aligned}
$$

This decomposition is experimentally possible provided that the height-height correlations are short-ranged and the inverse correlation length $\xi^{-1}$ is significantly greater than the typical resolution $q_{x y \text {, min }}\left(\sim \Delta q_{y}=10^{-5}-10^{-4} \AA^{-1}\right)$. For $\xi^{-1}$ $\gg q_{x y \text {, min }}$, the surface diffuse scattering intensity, represented by the second (integral) term in Eq. (A1), is usually both small and smoothly varying at $q_{x y}=0$ and can easily be separated empirically from the specular reflection [the first term in Eq. (A1)] [71]. Thus, with $\Xi\left(\mathbf{q}_{x y}=0\right)=1$, the principal effect of surface roughness is to reduce the magnitude of specular reflectivity $R\left(q_{z}\right)$ from the flat surface values through Debye-Waller (DW) factors,

$$
R_{i j}\left(0, q_{z}\right) \approx e^{-(1 / 2)\left[\left\langle h_{i}^{2}(0)\right\rangle+\left\langle\left\langle h_{j}^{2}(0)\right\rangle\right] q_{z}^{2}\right.} .
$$

By contrast, the decomposition into true specular and diffuse terms in Eq. (A1) cannot be directly applied to liquid surfaces because the presence of thermal capillary waves causes the height-height correlations to be quasi-long-ranged $[29,35,72]$. For simple surfaces with either a single interface or perfectly conformal roughness, the characteristic features of liquid surface scattering can be summarized as follows [30]. For given surface tension $\gamma$ and liquid mass density $\rho_{m}$, the height-height correlations are limited by the gravitational capillary length $\xi_{g}=\sqrt{\gamma / \rho_{m} g}$, which is of the order of mm for typical liquids, i.e., $\xi_{g}^{1} \ll q_{x y \text {,min }}$ [73]. Moreover, because of the logarithmic behavior of $g\left(r_{x y}\right) \equiv\left\langle\left[h\left(r_{x y}\right)-h(0)\right]^{2}\right\rangle$ $\sim 2\left(k_{B} T / 2 \pi \gamma\right) \ln \left(r_{x y}\right) \quad$ for $\quad q_{\max }^{-1}(\sim \AA) \ll r_{x y} \ll \xi_{g}(\sim \mathrm{mm})$ $[28,30]$, thermal diffuse scattering (TDS) from liquid surfaces displays an algebraic, cusplike singularity $d \sigma / d \Omega$ $\sim 1 / q_{x y}^{2-\eta}$ with $\eta \equiv\left(k_{B} T / 2 \pi \gamma\right) q_{z}^{2}[28,30]$ that is only truncated at $q_{x y} \sim \xi_{g}^{1}$. Since the resolution widths $\left(\Delta q_{x}, \Delta q_{y}\right)$ of virtually all practical $\mathrm{x}$-ray reflectometers are large compared to $\xi_{g}{ }^{1}$, distances of order $\xi_{g}$ cannot be resolved $[31,71,73]$. Consequently, for liquid surfaces there is no practical way to make a simple distinction between the true specular signal and the small-angle diffuse scattering that is sharply peaked at $q_{x y}=0$.

The above problem can be circumvented by recognizing that it is not necessary to consider the detailed behavior of $g\left(r_{x y}\right)$ for the large values of $r_{x y}\left(\gg 1 / q_{x y, \min } \sim 1 / \Delta q_{y}\right)$ that cannot be resolved experimentally $[30,46,47]$. One approach that has proven to be successful is (i) to assume the singular form $d \sigma / d \Omega \sim N / q_{x y}^{2-\eta}$ over the entire range $0<q_{x y}<q_{\max }$ by taking the limit $\xi_{g} \rightarrow \infty$; and (ii) to obtain the proper normalization factor $N$ by applying an appropriate sum rule. Step (ii) is equivalent to setting

$$
\frac{1}{2} g\left(r_{x y}\right) \approx \frac{k_{B} T}{2 \pi \gamma} \ln \left(q_{\max } r_{x y}\right) .
$$

The resulting single-interface analogue of $R_{i j}$ is approximately given by $[30,46,47]$

$$
R_{1}\left(\mathbf{q}_{x y}, q_{z}\right) \approx \int d^{2} \mathbf{q}_{x y}^{\prime} \Xi\left(\mathbf{q}_{x y}-\mathbf{q}_{x y}^{\prime}\right) \frac{2 \pi \eta}{q_{x y}^{\prime 2}}\left(\frac{q_{x y}^{\prime}}{q_{\max }}\right)^{\eta} .
$$

This single expression applies to both XR $\left(\mathbf{q}_{x y}=0\right)$ and XDS $\left(\mathbf{q}_{x y} \neq 0\right)$. The underlying assumption is that for all practical purposes, the measured intensity, whether specular or offspecular, corresponds to the integration of TDS over the resolution. For $q_{x y}$ that are small compared to the reciprocal of the molecular lengths, this assumption follows from general hydrodynamic considerations [74]. Empirically, the quantitative accuracy of Eq. (A4) is supported by the XR/ XDS measurements on various simple, laterally uniform liquid surfaces [46-51].

One important implication of Eq. (A4) is that even for $\mathrm{XR}, R_{1}\left(0, q_{z}\right)$ is resolution-dependent. Nevertheless, as shown previously, it is often possible to make a DW-like approximation with an effective capillary-wave roughness $\sigma_{c}$ $[30,31,33,47]$,

$$
R_{1}\left(0, q_{z}\right) \approx e^{-\sigma_{c}^{2} q_{z}^{2}}
$$

In contrast to the solid case [Eq. (A2)], however, the measured roughness $\sigma_{c}$ for liquids depends on the resolution $[31,33,34,38]$ through $\Xi\left(\mathbf{q}_{x y}\right)$ in Eq. (A4). For the resolution function that is rectangular in $q_{y}$ and infinitely wide along $q_{x}$ 
[Eq. (34)], Eq. (A5) can be shown to be correct to order $q_{z}^{2}$ with $[31,34,38]$

$$
\sigma_{c}^{2} \approx \frac{k_{B} T}{2 \pi \gamma} \ln \left(\frac{q_{\max }}{\Delta q_{y}}\right) .
$$

This apparent roughness $\sigma_{c}$ for liquid surfaces is smaller than the root-mean-square amplitude $\left\langle h^{2}(0)\right\rangle^{1 / 2}$ of height fluctuations, where

$$
\left\langle h^{2}(0)\right\rangle \approx \frac{k_{B} T}{2 \pi \gamma} \ln \left(q_{\max } \xi_{g}\right)
$$

for capillary waves on bulk liquid surfaces $\left(\Delta q_{y} \gg \xi_{g}^{1}\right)$ [31,36]. Moreover, the relation $\Delta q_{y} \approx k \sin (\beta) \Delta \beta$ $=\left(q_{z} / 2\right) \Delta \beta$ at the specular condition $(\alpha=\beta)$ implies that for typical XR measurements with fixed angular resolution $\Delta \beta$, $\sigma_{c}$ should in principle display a weak logarithmic dependence on $q_{z}$ through $\Delta q_{y}[31,34]$.

In order to extract the intrinsic profile of liquid surfaces from XR, these subtle effects of small-angle TDS on the apparent measured reflectivity need to be properly accounted for [30]. This requires prior knowledge of parameters such as the experimental resolutions, $T$ and $\gamma$. If one of these parameters is unknown or involves large uncertainties, simultaneous measurements of XR and XDS are necessary in order to quantitatively separate the intrinsic form factor $\left|\Theta\left(q_{z}\right)\right|^{2}$ from the DW factor $R_{1}\left(0, q_{z}\right)$. In Sec. III approximations similar to those that give rise to Eqs. (A4)-(A6) are developed for liquid surfaces with two interacting fluid-fluid interfaces.

\section{APPENDIX B: APPLICABILITY OF ANALYTIC APPROXIMATION FOR XR}

The two methods described in Sec. III D for calculating reflectivity are compared quantitatively in Fig. 8. In the plot of $R / R_{F}$ in Fig. 8(a), the open circles represent the numerical integration method [Eq. (6) with Eq. (35)], and the solid line corresponds to the analytic small- $q_{z}$ approximation with the DW factors [Eq. (41) with Eqs. (38)-(40)]. The calculations are based on the parameters for a PFMC film on C20 and the resolution used in the actual XR measurements. The period of the interference (Kiessig) fringes is a measure of the layer thickness $(D=100 \AA)$, and the decay rates for both the overall magnitude and the oscillation amplitude depend on the various height fluctuations. The solid lines in Figs. 8(b)-8(e) represent the relative differences, $\Delta R / R$ or $\Delta R_{i j} / R_{i j}$, between the numerical integration and the DW method, where each difference has been normalized to the numerically integrated value; the dashed curves in Figs. 8(b) and 8(e) show the same except that we set $\Delta_{c, 12}=0$ for the small- $q_{z}$ approximation. Also plotted in Figs. 8(c)-8(e) (dotted lines) is the $q_{z}$ dependence of the exponents $\eta_{i}$ and $\eta_{12}$ [Eq. (28)]. The inequality $\eta_{1}>\eta_{2}>\eta_{12}$ at any given $q_{z}$ originates from the fact that the surface tension of the liquid-liquid interface $[i$ $=1$, Fig. 1(b)] is lower than that of the liquid-vapor interface $(i=2)$, i.e., $\gamma_{1}<\gamma_{2}<\gamma_{1}+\gamma_{2}$ (Table I).

According to Fig. 8 , the DW representation of $R / R_{F}$ [Eq. (41)] should provide an accurate description of the reflectiv-

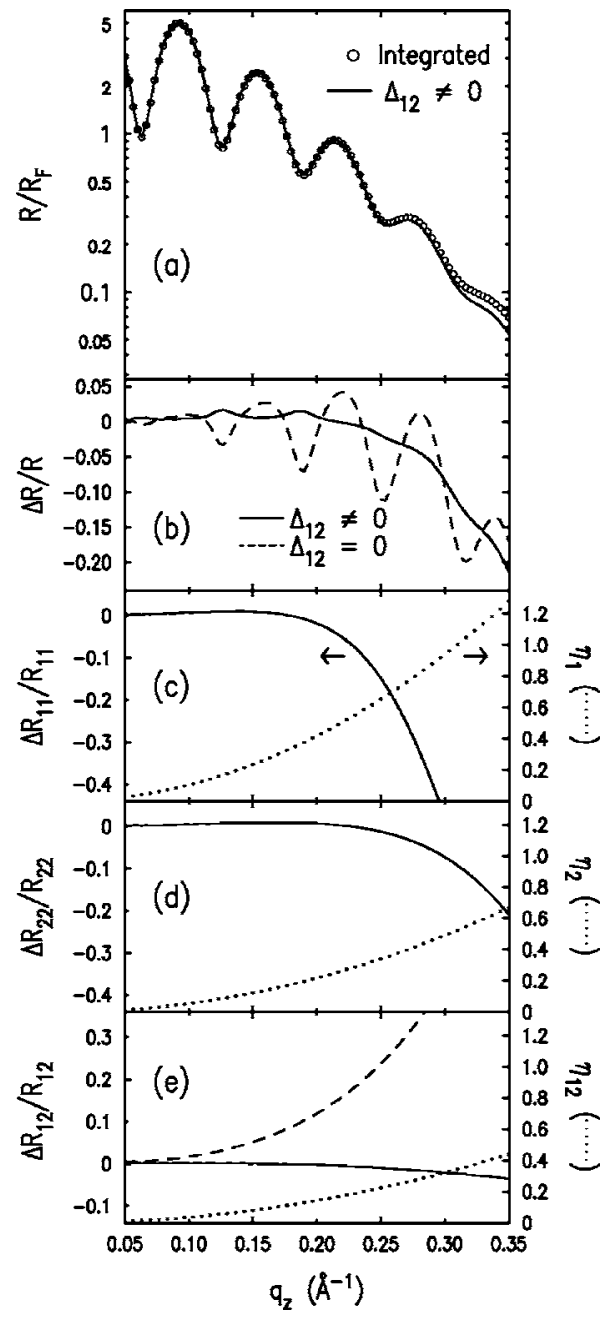

FIG. 8. (a) Theoretical Fresnel-normalized specular reflectivity $R / R_{F}$ vs $q_{z}$, calculated using the parameters in Table I for a PFMC wetting film on $\mathrm{C} 20$ at $37^{\circ} \mathrm{C}$ and $D=100 \AA$, the rectangular FWHM resolution $\Delta q_{y}=\left(q_{z} / 2\right) \Delta \beta$ of the XR experiment, and zero intrinsic widths $w_{i}=0$ for simplicity. The symbols $(\bigcirc)$ represent the calculation in which the factors $R_{i j}\left(0, q_{z}\right)$ are evaluated through numerical integration [Eqs. (6) and (35)]. The solid curve is based on the analytic form of $R / R_{F}$ [Eq. (41)] for which $R_{i j}\left(0, q_{z}\right)$ are approximated by Debye-Waller (DW) factors with effective capillary roughness parameters $\sigma_{c, i i}$ [Eqs. (36) and (38)-(40)]; the calculation accounts for the interfacial correlation-correction term $\left(\Delta_{c, 12} \neq 0\right)$ in Eq. (41). The relative difference between the numerical integration method and the DW factor method is shown as the solid curve for (b) $R\left(q_{z}\right)$, (c) $R_{11}\left(0, q_{z}\right)$, (d) $R_{22}\left(0, q_{z}\right)$, and (e) $R_{12}\left(0, q_{z}\right)$. The dashed curves in (b) and (e) represent, respectively, the relative difference in $R$ and in $R_{12}$ that would result if the correlation correction for the DW approach were neglected, i.e., if $\Delta_{c, 12}=0$. The dotted curves in (c), (d), and (e) show the $q_{z}$ dependence of exponents $\eta_{1}, \eta_{2}$, and $\eta_{12}$, respectively [Eq. (28)].

ity for the PFMC film on C20 up to $q_{z} \sim 0.3 \AA^{-1}(\Delta R / R$ $\sim 5 \%)$, provided that the $\Delta_{c, 12}$-dependent term is properly accounted for. If this term were neglected, the error in the oscillation amplitude of $R / R_{F}$ would become significant at even smaller $q_{z}$ [Figs. 8(b) and 8(e)]. Another more general observation is that the DW representation of $R_{i j}\left(0, q_{z}\right)$ is ac- 
curate so long as the associated exponent $\eta\left(=\eta_{i}\right.$ or $\left.\eta_{12}\right) \ll 1$. In particular, the condition $\eta_{i} \sim 1$ for the larger of the two exponents $\eta_{i}$ can be used to set an upper limit on $q_{z}$ above which the DW approximation of $R / R_{F}$ should be considered unreliable [see Figs. 8(b) and 8(c)]. In this regard, it is to be noted that for the TDS from the simple liquid surface (Appendix A), the integration of $d \sigma / d \Omega \sim 1 / q_{x y}^{2-\eta}$ over the infinitely wide resolution in $q_{x}$ causes the scattered intensity to display a singularity of form $\sim 1 / q_{y}^{1-\eta}[29,71]$. As pointed out previously [30,49,51], this singularity vanishes as $\eta \rightarrow 1$, and the distinction between surface and bulk diffuse scattering becomes meaningless for $\eta>1$.

\section{APPENDIX C: XR FOR GAUSSIAN AND ERROR- FUNCTION-BASED RESOLUTION FUNCTIONS}

In Sec. III D specular reflectivity is calculated using the rectangular resolution function [see Eqs. (34) and (35)], due to the relatively wide detector resolution used in the present XR measurements. We summarize here the results of equivalent calculations for two other resolution functions that may be more appropriate for narrower resolutions than used in this study. As before, the conditions $q_{\max }^{-1} \ll \xi \ll \xi_{g}$ and $\Delta q_{y}$ $\gg \xi_{g}^{1}$ are assumed to hold below.

For the Gaussian resolution function [Eq. (29)], the factors $R_{i j}\left(0, q_{z}\right)$ for XR can be obtained by setting $q_{y}=0$ in Eq. (30) and using the approximate correlation functions of form Eqs. (26) and (27). The results can be expressed in terms of analytic functions as follows:

$$
\begin{aligned}
R_{i i}\left(0, q_{z}\right) \approx & \operatorname{erf}\left(\frac{\Delta q_{y} / q_{\max }}{2 \sqrt{\pi}}\right) \\
& +\frac{\Delta q_{y} / q_{\max }}{2 \pi}\left\{\Gamma\left(\frac{1-\eta_{i}}{2}, \frac{\left(\Delta q_{y} / q_{\max }\right)^{2}}{4 \pi}\right)\right. \\
& \left.-\frac{1}{\left(q_{\max } \xi\right)^{1-\eta_{i}}} \Gamma\left(\frac{1-\eta_{i}}{2}, \frac{\left(\Delta q_{y} \xi\right)^{2}}{4 \pi}\right)\right\} \\
& +\frac{\Delta q_{y} \xi}{2 \pi\left(q_{\max } \xi\right)^{\eta_{i}}} \Gamma\left(\frac{1-\eta_{12}}{2}, \frac{\left(\Delta q_{y} \xi\right)^{2}}{4 \pi}\right),
\end{aligned}
$$

$$
\begin{aligned}
R_{12}\left(0, q_{z}\right) \approx & \left(q_{\max } \xi\right)^{-(1 / 2)\left(\eta_{1}+\eta_{2}\right)}\left\{\operatorname{erf}\left(\frac{\Delta q_{y} \xi}{2 \sqrt{\pi}}\right)\right. \\
& \left.+\frac{\Delta q_{y} \xi}{2 \pi} \Gamma\left(\frac{1-\eta_{12}}{2}, \frac{\left(\Delta q_{y} \xi\right)^{2}}{4 \pi}\right)\right\},
\end{aligned}
$$

where $\Gamma(\alpha, x)$ is the (upper) incomplete Gamma function. Note that the $q_{z}$ dependence of $R_{i j}\left(0, q_{z}\right)$ is buried in the parameters $\left\{\eta_{1}, \eta_{2}, \eta_{12}, \Delta q_{y}\right\}$.

Alternatively, for the $q_{y}$ resolution that consists of a combination of error functions, i.e.,

$$
\Xi\left(q_{y}\right)=\frac{1}{2}\left\{\operatorname{erf}\left(\frac{q_{y}+\Delta q_{y} / 2}{\sqrt{2} \delta}\right)-\operatorname{erf}\left(\frac{q_{y}-\Delta q_{y} / 2}{\sqrt{2} \delta}\right)\right\},
$$

one obtains

$$
R_{i j}\left(0, q_{z}\right)=\frac{\Delta q_{y}}{\pi} \int_{0}^{\infty} d y \frac{\sin \left(\Delta q_{y} y / 2\right)}{\Delta q_{y} y / 2} e^{-\delta^{2} y^{2} / 2} e^{-(1 / 2) g_{i j}(y) q_{z}^{2}} .
$$

In the limit of $\delta \rightarrow 0$, Eqs. (C3) and (C4) reduce to the forms for the rectangular resolution, i.e., Eqs. (34) and (35), respectively. The integration in Eq. (C4) can be carried out numerically and should converge faster than for the rectangular resolution due to the presence of the factor $e^{-\delta^{2} y^{2} / 2}$ in the integrand.

It should be noted that the above expressions for $R_{i j}\left(0, q_{z}\right)$ are not particularly suited for understanding the qualitative behavior of specular reflectivity and its dependence on various parameters. This disadvantage can be overcome for small $q_{z}$ by approximating Eqs. (C1), (C2), and (C4) with the Debye-Waller factors, as discussed in Sec. III D.
[1] J. Als-Nielsen and D. McMorrow, Elements of Modern X-ray Physics (Wiley, Chichester, 2001).

[2] S. K. Sinha, Curr. Opin. Solid State Mater. Sci. 1, 645 (1996).

[3] E. Chason and T. M. Mayer, Crit. Rev. Solid State Mater. Sci. 22, 1 (1997).

[4] M. Tolan, X-ray Scattering from Soft-Matter Thin Films: Material Science and Basic Research (Springer-Verlag, New York, 1999).

[5] E. E. Fullerton, J. Pearson, C. H. Sowers, S. D. Bader, X. Z. Wu, and S. K. Sinha, Phys. Rev. B 48, 17432 (1993).

[6] S. K. Sinha, M. K. Sanyal, S. K. Satija, C. F. Majkrzak, D. A. Neumann, H. Homma, S. Szpala, A. Gibaud, and H. Morkoc, Physica B 198, 72 (1994).
[7] J.-P. Schlomka, M. Tolan, L. Schwalowsky, O. H. Seeck, J. Stettner, and W. Press, Phys. Rev. B 51, 2311 (1995).

[8] J. Stettner, L. Schwalowsky, O. H. Seeck, M. Tolan, W. Press, C. Schwarz, and H. v. Kanel, Phys. Rev. B 53, 1398 (1996).

[9] M. Lutt, J.-P. Schlomka, M. Tolan, J. Stettner, O. H. Seeck, and W. Press, Phys. Rev. B 56, 4085 (1997).

[10] I. M. Tidswell, T. A. Rabedeau, P. S. Pershan, and S. D. Kosowsky, Phys. Rev. Lett. 66, 2108 (1991).

[11] O. H. Seeck, P. Muller-Buschbaum, M. Tolan, and W. Press, Europhys. Lett. 29, 699 (1995).

[12] A. K. Doerr, M. Tolan, W. Prange, J.-P. Schlomka, T. Seydel, W. Press, D. Smilgies, and B. Struth, Phys. Rev. Lett. 83, 3470 (1999). 
[13] W. Zhao, M. H. Rafailovich, J. Sokolov, L. J. Fetters, R. Plano, M. K. Sanyal, S. K. Sinha, and B. B. Sauer, Phys. Rev. Lett. 70, 1453 (1993).

[14] M. Tolan, O. H. Seeck, J.-P. Schlomka, W. Press, J. Wang, S. K. Sinha, Z. Li, M. H. Rafailovich, and J. Sokolov, Phys. Rev. Lett. 81, 2731 (1998).

[15] J. Wang, M. Tolan, O. H. Seeck, S. K. Sinha, O. Bahr, M. H. Rafailovich, and J. Sokolov, Phys. Rev. Lett. 83, 564 (1999).

[16] Y.-S. Seo, T. Koga, J. Sokolov, M. H. Rafailovich, M. Tolan, and S. Sinha, Phys. Rev. Lett. 94, 157802 (2005).

[17] A. Gibaud, N. Cowlam, G. Vignaud, and T. Richardson, Phys. Rev. Lett. 74, 3205 (1995).

[18] V. Nitz, M. Tolan, J.-P. Schlomka, O. H. Seeck, J. Stettner, W. Press, M. Stelzle, and E. Sackmann, Phys. Rev. B 54, 5038 (1996).

[19] J. K. Basu and M. K. Sanyal, Phys. Rev. Lett. 79, 4617 (1997).

[20] J. K. Basu, S. Hazra, and M. K. Sanyal, Phys. Rev. Lett. 82, 4675 (1999).

[21] J. Daillant and O. Belorgey, J. Chem. Phys. 97, 5837 (1992).

[22] M. Li, A. M. Tikhonov, D. J. Chaiko, and M. L. Schlossman, Phys. Rev. Lett. 86, 5934 (2001).

[23] M. Paulus, C. Gutt, and M. Tolan, Phys. Rev. E 72, 061601 (2005).

[24] J. Daillant and O. Belorgey, J. Chem. Phys. 97, 5824 (1992).

[25] M. L. Schlossman, M. Li, D. M. Mitrinovic, and A. M. Tikhonov, High Perform. Polym. 12, 551 (2000).

[26] M. Li and M. L. Schlossman, Phys. Rev. E 65, 061608 (2002).

[27] K. Shin, Y. Pu, M. H. Rafailovich, J. Sokolov, O. H. Seeck, S. K. Sinha, M. Tolan, and R. Kolb, Macromolecules 34, 5620 (2001).

[28] S. K. Sinha, E. B. Sirota, S. Garoff, and H. B. Stanley, Phys. Rev. B 38, 2297 (1988).

[29] M. K. Sanyal, S. K. Sinha, K. G. Huang, and B. M. Ocko, Phys. Rev. Lett. 66, 628 (1991).

[30] P. S. Pershan, Colloids Surf., A 171, 149 (2000).

[31] A. Braslau, P. S. Pershan, G. Swislow, B. M. Ocko, and J. Als-Nielsen, Phys. Rev. A 38, 2457 (1988).

[32] J. Daillant, L. Bosio, J. J. Benattar, and J. Meunier, Europhys. Lett. 8, 453 (1989).

[33] D. K. Schwartz, M. L. Schlossman, E. H. Kawamoto, G. J. Kellogg, P. S. Pershan, and B. M. Ocko, Phys. Rev. A 41, 5687 (1990).

[34] B. M. Ocko, X. Z. Wu, E. B. Sirota, S. K. Sinha, and M. Deutsch, Phys. Rev. Lett. 72, 242 (1994).

[35] J. D. Weeks, J. Chem. Phys. 67, 3106 (1977).

[36] M. P. Gelfand and M. E. Fisher, Physica A 166, 1 (1990).

[37] M. Sferrazza, C. Xiao, R. A. L. Jones, D. G. Bucknall, J. Webster, and J. Penfold, Phys. Rev. Lett. 78, 3693 (1997).

[38] D. M. Mitrinovic, A. M. Tikhonov, M. Li, Z. Huang, and M. L. Schlossman, Phys. Rev. Lett. 85, 582 (2000).

[39] An alternative approach in which the surface tension for capillary waves is assumed to depend on the lateral wave vector has been suggested previously; see, e.g., A. K. Doerr et al., Phys. Rev. Lett. 83, 3470 (1999), and references therein.

[40] O. Gang, M. Fukuto, P. Huber, and P. S. Pershan, Colloids Surf., A 206, 293 (2002).

[41] V. Holy, J. Kubena, I. Ohlidal, K. Lischka, and W. Plotz, Phys. Rev. B 47, 15896 (1993).

[42] V. Holy and T. Baumbach, Phys. Rev. B 49, 10668 (1994).

[43] S. K. Sinha, J. Phys. III 4, 1543 (1994).
[44] J. Daillant, K. Quinn, C. Gourier, and F. Rieutord, J. Chem. Soc., Faraday Trans. 92, 505 (1996).

[45] J. Daillant, S. Mora, C. Fradin, M. Alba, A. Braslau, and D. Luzet, Appl. Surf. Sci. 182, 223 (2001).

[46] M. Fukuto, R. K. Heilmann, P. S. Pershan, J. A. Griffiths, S. M. Yu, and D. A. Tirrell, Phys. Rev. Lett. 81, 3455 (1998).

[47] H. Tostmann, E. DiMasi, P. S. Pershan, B. M. Ocko, O. G. Shpyrko, and M. Deutsch, Phys. Rev. B 59, 783 (1999).

[48] M. Fukuto, R. K. Heilmann, P. S. Pershan, S. M. Yu, J. A. Griffiths, and D. A. Tirrell, J. Chem. Phys. 111, 9761 (1999).

[49] O. Shpyrko, P. Huber, A. Grigoriev, P. Pershan, B. Ocko, H. Tostmann, and M. Deutsch, Phys. Rev. B 67, 115405 (2003).

[50] M. Fukuto, R. K. Heilmann, P. S. Pershan, A. Badia, and R. B. Lennox, J. Chem. Phys. 120, 3446 (2004).

[51] O. Shpyrko, M. Fukuto, P. Pershan, B. Ocko, I. Kuzmenko, T. Gog, and M. Deutsch, Phys. Rev. B 69, 245423 (2004).

[52] B. R. McClain, M. Yoon, J. D. Litster, and S. G. J. Mochrie, Eur. Phys. J. B 10, 45 (1999).

[53] D. M. Mitrinovic, S. M. Williams, and M. L. Schlossman, Phys. Rev. E 63, 021601 (2001).

[54] S. Dietrich, in Phase Transitions and Critical Phenomena, edited by C. Domb and J. L. Lebowitz (Academic, New York, 1988), Vol. 12, p. 1.

[55] B. M. Law, Prog. Surf. Sci. 66, 159 (2001).

[56] P. G. de Gennes, Rev. Mod. Phys. 57, 827 (1985).

[57] D. E. Sullivan and M. M. Telo da Gama, in Fluid Interfacial Phenomena, edited by C. A. Croxton (Wiley, New York, 1986), p. 45.

[58] J. Israelachvili, Intermolecular and Surface Forces (Academic, London, 1992).

[59] D. Andelman, J.-F. Joanny, and M. O. Robbins, Europhys. Lett. 7, 731 (1988).

[60] M. O. Robbins, D. Andelman, and J.-F. Joanny, Phys. Rev. A 43, 4344 (1991).

[61] The limiting forms in Eqs. (22)-(25) can be obtained by replacing the Bessel function $J_{0}\left(q_{x y} r_{x y}\right)$ in the integrands of Eqs. (9)-(11) with a step function that is unity for $q_{x y}<r_{x y}^{-1}$ and zero for $q_{x y}>r_{x y}^{-1}$.

[62] D. Beysens and M. Robert, J. Chem. Phys. 87, 3056 (1987).

[63] B. M. Ocko, X. Z. Wu, E. B. Sirota, S. K. Sinha, O. Gang, and M. Deutsch, Phys. Rev. E 55, 3164 (1997).

[64] This can be seen from the fact that $d \sigma / d \Omega \sim f\left(q_{x y}, q_{z}\right) / \sin (\alpha)$ for laterally isotropic surfaces and $\Delta \Omega=\cos (\beta) \Delta \psi \Delta \beta$ $\sim \Delta \psi \Delta \beta$ for $\beta \ll 1$.

[65] The macroscopic surface tension of the liquid-liquid interface between bulk PFMC and bulk C20 has been measured by C. Rulison of Kruss USA, using the pendant drop method and the same chemical materials as used in this study.

[66] R. K. Heilmann, M. Fukuto, and P. S. Pershan, Phys. Rev. B 63, 205405 (2001).

[67] M. Fukuto, Y. F. Yano, and P. S. Pershan, Phys. Rev. Lett. 94, 135702 (2005).

[68] L. G. Parratt, Phys. Rev. 95, 359 (1954).

[69] J. Lekner, Theory of Reflection (Martin Nijhoff, Dordrecht, 1987).

[70] The DW factor for the BA is usually expressed as $\exp ($ $-\sigma^{2} q_{z}^{2}$ ); however, as a result of refraction effects, the value of $q_{z}$ in the material should actually be given by $q_{z}=\left(q_{z, 0}^{2}\right.$ $\left.-q_{c}^{2}\right)^{1 / 2}$, where $q_{z, 0}=2 k \sin (\alpha)$.

[71] S. K. Sinha, Physica B 173, 25 (1991). 
[72] M. S. Wertheim, J. Chem. Phys. 65, 2377 (1976).

[73] Note that for a liquid-liquid interface with extremely low surface tension, the capillary length $\xi_{g}$ can be much smaller than $\sim$ mm such that the inverse $\xi_{g}$ is comparable to the lateral wave-vector resolution $\Delta q_{x y}$. See, e.g., B. R. McClain et al.,
Eur. Phys. J. B 10, 45 (1999); D. M. Mitrinovic, S. M. Williams, and M. L. Schlossman, Phys. Rev. E 63, 021601 (2001).

[74] D. Forster, Hydrodynamic Fluctuations, Broken Symmetry, and Correlation Functions (W.A. Benjamin, Reading, MA, 1975). 